\title{
Nutraceutical support in heart failure: a position paper of the International Lipid Expert Panel (ILEP)
}

Arrigo F. G. Cicero ${ }^{1 *}$, Alessandro Colletti ${ }^{2}$, Stephan von Haehling ${ }^{3}$, Dragos Vinereanu ${ }^{4}$, Agata Bielecka-Dabrowa ${ }^{5}$, Amirhossein Sahebkar ${ }^{6,7,8}$, Peter P. Toth ${ }^{9}$, Željko Reiner ${ }^{10}$, Nathan D. Wong ${ }^{11}$, Dimitri P. Mikhailidis ${ }^{12}$, Claudio Ferri ${ }^{13}$ and Maciej Banach ${ }^{14,15,16 *}$ on behalf of the International Lipid Expert Panel $\dagger$

${ }^{1}$ Department of Medical and Surgical Sciences, University of Bologna, Bologna, Italy

${ }^{2}$ Department of Science and Drug Technology, University of Turin, Turin, Italy

${ }^{3}$ Department of Cardiology and Pneumology, University of Goettingen Medical Center, Goettingen, Germany; German Center for Cardiovascular Disorders (DZHK), partner site Goettingen, Germany

${ }^{4}$ University of Medicine and Pharmacy Carol Davila, University and Emergency Hospital, Bucharest, Romania

${ }^{5}$ Department of Cardiology and Congenital Diseases of Adults, Polish Mother's Memorial Hospital Research Institute (PMMHRI),

Lodz, Poland

${ }^{6}$ Halal Research Center of IRI, FDA, Tehran, Iran

${ }^{7}$ Biotechnology Research Center, Pharmaceutical Technology Institute, Mashhad University of Medical Sciences, Mashhad, Iran

${ }^{8}$ Neurogenic Inflammation Research Center, Mashhad University of Medical Sciences, Mashhad, Iran

${ }^{9}$ Ciccarone Center for Prevention of Heart Disease, Division of Cardiology, Department of Medicine, Johns Hopkins University School of Medicine, Baltimore, MD, USA

${ }^{10}$ Department for Metabolic Diseases, University Hospital Center Zagreb, School of Medicine, Zagreb University, Zagreb, Croatia

${ }^{11}$ Heart Disease Prevention Program, Division of Cardiology, University of California, Irvine, CA, USA

${ }^{12}$ Department of Clinical Biochemistry, Royal Free Campus, University College London Medical School, University College

London, London, UK

${ }^{13}$ Department of Life, Health and Environmental Sciences, University of L'Aquila, Coppito, L'Aquila, Italy

${ }^{14}$ Department of Hypertension, Medical University of Lodz, Lodz, Poland

${ }^{15}$ Polish Mother's Memorial Hospital Research Institute (PMMHRI), Lodz, Poland

${ }^{16}$ Cardiovascular Research Centre, University of Zielona Gora, Zielona Gora, Poland

\section{Abstract}

Heart failure (HF) is a complex clinical syndrome that represents a major cause of morbidity and mortality in Western countries. Several nutraceuticals have shown interesting clinical results in HF prevention as well as in the treatment of the early stages of the disease, alone or in combination with pharmacological therapy. The aim of the present expert opinion position paper is to summarise the available clinical evidence on the role of phytochemicals in HF prevention and/or treatment that might be considered in those patients not treated optimally as well as in those with low therapy adherence. The level of evidence and the strength of recommendation of particular HF treatment options were weighed up and graded according to predefined scales. A systematic search strategy was developed to identify trials in PubMed (January 1970 to June 2019). The terms 'nutraceuticals', 'dietary supplements', 'herbal drug' and 'heart failure' or 'left verntricular dysfunction' were used in the literature search. The experts discussed and agreed on the recommendation levels. Available clinical trials reported that the intake of some nutraceuticals (hawthorn, coenzyme $\mathrm{Q}_{10}$, L-carnitine, D-ribose, carnosine, vitamin $\mathrm{D}$, probiotics, $n$ - 3 PUFA and beet nitrates) might

Abbreviations: 25(OH)D, 25-hydroxyvitamin D; 6MWT, 6-min walking test; AA, amino acid; BNP, brain natriuretic peptide; BRJ, beetroot juice; CoQ10, coenzyme $\mathrm{Q}_{10}$; E, early diastolic filling velocity; E', early annular mitral velocity; EF, ejection fraction; HF, heart failure; HFDC, high-flavanol dark chocolate; HFmrEF, heart failure with mid-range ejection fraction; HFpEF, heart failure with preserved ejection fraction; HFrEF, heart failure with reduced ejection fraction; HR, hazard ratio; hsCRP, high-sensitivity C-reactive protein; LFDC, low-flavanol dark chocolate; LV, left ventricular; $\mathrm{LVEF}$, left ventricular ejection fraction; $\mathrm{NO}_{3}-$, inorganic nitrate; NT-proBNP, N-terminal pro-B-type natriuretic peptide; NYHA, New York Heart Association; QoL, quality of life; Q-SYMBIO, Q10-SYMptoms, BIomarker status [Brain-Natriuretic Peptide], and long-term Outcome [hospitalizations/mortality]; RCT, randomised clinical trial; SMD, standardised mean difference; SPICE, Survival and Prognosis: Investigation of Crataegus Extract WS 1442 in congestive heart failure; TMAO, trimethylamine $N$-oxide; vitD, vitamin $\mathrm{D}_{2}$ and $\mathrm{D}_{3}$; WMD, weighted mean difference.

* Corresponding authors: Professor Arrigo F. G. Cicero, fax +39 51391320, email arrigo.cicero@unibo.it; Professor Maciej Banach, fax +48 4227115 60, email maciejbanach77@gmail.com

$\dagger$ For the list of the International Lipid Expert Panel Experts, see the Appendix. 
be associated with improvements in self-perceived quality of life and/or functional parameters such as left ventricular ejection fraction, stroke volume and cardiac output in HF patients, with minimal or no side effects. Those benefits tended to be greater in earlier HF stages. Available clinical evidence supports the usefulness of supplementation with some nutraceuticals to improve HF management in addition to evidence-based pharmacological therapy.

Key words: Heart failure: Nutraceuticals: Coenzyme $Q_{10}$ : Herbal drugs: Nutritional supplements

(Received 22 November 2019; revised 19 January 2020; accepted 24 January 2020)

\section{Introduction}

Heart failure (HF) prevalence in the USA is about 5 million individuals $^{(1)}$, while it affects more than 23 million individuals worldwide $^{(2)}$. HF is the main factor responsible for hospitalisation and disability in the elderly and is the cause of one in nine deaths in the USA ${ }^{(3)}$. Consequently, HF imposes a great problem to the healthcare system, amounting to more than $\$ 39$ billion annually in the $\mathrm{USA}^{(3)}$. In Europe, the prevalence and incidence of $\mathrm{HF}$, and related costs, are quite similar ${ }^{(4,5)}$. Even though there have been relevant improvements in HF prevention and treatment, quality of life (QoL) is often impaired and mortality rates are greater than $10 \%$ per year, reaching $20-50 \%$ in more advanced disease $^{(6)}$.

$\mathrm{HF}$ is attributed mainly to four underlying conditions: hypertension, coronary artery disease, cardiomyopathy and valvular heart disease ${ }^{(1,2)}$; however, genetic causes, particularly in dilated cardiomyopathy, also play an immense role ${ }^{(7)}$. Still, HF could be partly prevented by improving lifestyle, while an improvement in lifestyle is also suggested when HF has already been diagnosed ${ }^{(8,9)}$. Therapeutic lifestyle changes include adherence to a Mediterranean diet, a reduced-Na diet or Dietary Approaches to Stop Hypertension (DASH) diet as well as exercise training ${ }^{(10,11)}$, which are able to reduce the risk of developing $\mathrm{HF}$ and improve endothelial function, exercise capacity and QoL in HF patients ${ }^{(12)}$. In particular, the DASH diet can protect against HF risk by as much as $29 \%{ }^{(13)}$. Moreover, weight loss, moderation of alcohol consumption and increased aerobic exercise are recommended measures $^{(14)}$.

In recent years, epidemiological studies and clinical trials have investigated the possibility that some dietary supplements and phytochemicals (overall referred to as natural products or nutraceuticals) can contribute to the improvement of HF-related symptoms (Fig. 1).

The aim of this position paper is to provide, for the first time, physicians and nutrition experts with a practical tool presenting the scientific evidence on efficacy and safety of nutraceuticals, eventually supporting their use as further HF symptoms improvers, as an add-on to optimal pharmacological treatment.

\section{Methods}

A systematic search strategy was developed to identify randomised clinical trials (RCT) and their meta-analyses in Medline/ PubMed (January 1970 to June 2019). The terms 'nutraceuticals', 'dietary supplements', 'herbal drug' and 'heart failure' were incorporated into an electronic search strategy. The experts discussed and agreed on the recommended levels. For each selected nutraceutical, a short description of the mechanism of action is reported, followed by the clinically observed effects and the most relevant tolerability notes.

The level of evidence of nutraceuticals tested on HF patients has been weighed up and graded according to predefined scales, as outlined in Table 1. Due to the fact of the limited data the experts did not decide to evaluate each selected nutraceutical with the class of the evidence. The experts of the writing and reviewing panels completed declaration of interest forms where real or potential sources of conflicts of interest might be perceived.

\section{Nutraceuticals}

Coenzyme $\mathrm{Q}_{10}$

Coenzyme $\mathrm{Q}_{10}$ (CoQ10) is an organic molecule, which is composed of a lipophilic core (benzoquinone) and an isoprenoid side chain; it was identified in 1940 and isolated for the first time from beef heart mitochondria by Frederick Crane of Wisconsin (USA), in $1957^{(15)}$. CoQ10 (10 refers to the number of isoprene repeats) is synthesised endogenously, starting from tyrosine and acetylcoenzyme A; it is universally present in the cells of the body, particularly concentrated in the mitochondria, in both reduced form (ubiquinol) and oxidised form (ubiquinone). The level of CoQ10 is highest in organs with high rates of metabolism such as the heart, kidney and liver $(114,66.5$ and 54.9 $\mu \mathrm{g} / \mathrm{g}$ tissue, respectively), where it functions as an energy transfer molecule $^{(16)}$. The overall body content of CoQ10 is only about 500-1500 mg and decreases with age. It is naturally contained in oily fish (such as salmon and tuna), organ meats (such as liver and heart) and whole grains, but it can be consumed also as a dietary supplement ${ }^{(17)}$.

By functioning as a reducing equivalent transfer agent between cytochromes in the mitochondrial electron transport chain, CoQ10 play a crucial role in oxidative phosphorylation (i.e. ATP biosynthesis). CoQ10 is also the only lipid-soluble antioxidant that slows lipid peroxidation in the circulation (by maintaining the reduced state of $\alpha$-tocopherol and ascorbic acid) ${ }^{(18)}$. Other functions of CoQ10 include stabilisation of Ca-dependent channels, cell signalling and cell growth through local regulation of cytosolic redox intermediates such as reduced nicotinamide adenine dinucleotide phosphate $(\mathrm{NADPH})^{(19)}$. Since CoQ10 is an essential cofactor for ATP biosynthesis, it is not surprising that the highest concentration, compared with other tissues, is focused in myocardial mitochondria ${ }^{(16)}$. Thus, it has been proposed that CoQ10 deficiency could play an aetiopathogenic role in the development and progression of HF.

CoQ10 bioavailability is extremely variable depending on dosage, particle size, formulation, the release method and the 
Table 1. Classification of the level of evidence

\begin{tabular}{ll}
\hline Level of evidence & Definition \\
\hline Level A & Data derived from multiple randomised clinical trials or their meta-analysis \\
Level B & Data derived from a single randomised clinical trial or large non-randomised studies \\
Level C & Consensus or opinion of experts and/or small studies, retrospective studies, registries \\
\hline
\end{tabular}

\begin{tabular}{|c|c|c|c|c|}
\hline \multicolumn{4}{|c|}{ Nutraceutical support to heart failure patients } & \multirow[b]{2}{*}{$\mathrm{Mg}$} \\
\hline Coenzyme $Q_{10}$ & Hawthorn & $n$-3 PUFA & L-Carnosine & \\
\hline $\begin{array}{l}\uparrow \text { ATP synthesis } \\
\mathrm{Ca}^{2+} \text { - channels } \\
\text { stabilisation } \\
\text { Antioxidant }\end{array}$ & $\begin{array}{l}\text { Anti-inflammatory } \\
\text { and inotropic effects } \\
\text { Coronary } \\
\text { vasodilatation }\end{array}$ & $\begin{array}{l}\text { Mitochondrial membrane } \\
\text { stabilisation } \\
\text { Anti-inflammatory effect } \\
\downarrow \text { Arterial stiffness }\end{array}$ & $\begin{array}{l}\text { Fe and } \mathrm{Cu} \text { chelation } \\
\text { Ca channels } \\
\text { sensitisation }\end{array}$ & $\begin{array}{l}\text { Haemodynamics and } \\
\text { electrophysiological } \\
\text { functioning }\end{array}$ \\
\hline Vitamin D & L-Carnitine & Probiotics & Vitamin $B_{1}$ & $\mathrm{Fe}$ \\
\hline $\begin{array}{l}\text { RAA system } \\
\text { modulation }\end{array}$ & $\begin{array}{l}\text { Antioxidant } \\
\beta \text {-Oxidation cofactor } \\
\uparrow \text { Endothelial } \\
\text { function }\end{array}$ & $\begin{array}{l}\downarrow \text { TMAO plasma levels } \\
\downarrow \text { Intestinal oedema, } \\
\text { inflammatory metabolites, } \\
\text { cardiac and vascular } \\
\text { remodelling }\end{array}$ & $\begin{array}{l}\text { Cofactor for ATP } \\
\text { metabolism } \\
\text { Coenzyme in the } \\
\text { pentose phosphate } \\
\text { pathway }\end{array}$ & $\begin{array}{l}\text { Component of myoglobin } \\
\text { and } \mathrm{Hb} \\
\uparrow \mathrm{Tissue} \text { oxygenation } \\
\text { Key component of } \\
\text { mitochondria }\end{array}$ \\
\hline D-Ribose & Beetroot & Cocoa and dark chocolate & Vitamin C & Vitamin E \\
\hline$\uparrow$ ATP synthesis & $\begin{array}{l}\uparrow \text { No production } \\
\uparrow \text { Endothelial } \\
\text { function }\end{array}$ & $\begin{array}{l}\text { Anti-inflammatory effect } \\
\downarrow \text { insulin resistance } \\
\downarrow \text { Arterial stiffness } \\
\text { Antioxidant effect }\end{array}$ & $\begin{array}{l}\text { Anti-inflammatory } \\
\text { Antioxidant effect } \\
\uparrow \text { Endothelial } \\
\text { function }\end{array}$ & $\begin{array}{l}\text { Antioxidant effect } \\
\downarrow \text { Arterial stiffness }\end{array}$ \\
\hline
\end{tabular}

Fig. 1. Nutraceutical support to heart failure patients. RAA, renin-angiotensin-aldosterone; TMAO, trimethylamine $\mathrm{N}$-oxide.

mode of administration (for example with or without water, before or after meals) ${ }^{(20)}$. In particular, ubiquinol (the reduced form) seems to be the most available form of CoQ10, especially if conveyed through micelles, liposomes, nano-emulsions, polymeric or solid lipid nanoparticles, solid and aqueous dispersions, or nanostructured lipid carriers supplemented in the fed state $^{(21,22)}$. However, because of its hydrophobicity and large molecular weight, absorption of dietary CoQ10 is slow and limited. The time taken to reach the maximum concentration $\left(\mathrm{T}_{\max }\right)$ is about $6 \mathrm{~h}$, with an elimination half-life of about $33 \mathrm{~h}$. The reference intervals for plasma CoQ10 range from 0.40 to $1.91 \mu \mathrm{mol} / 1$ in healthy adults ${ }^{(23,24)}$. Finally, in a recent paper by López-Lluch et $a l .{ }^{(25)}$, the level of bioavailability of seven different formulations of CoQ10 was measured over $48 \mathrm{~h}$ after ingestion of 100 $\mathrm{mg}$. The two best absorbable formulations were soft-gel capsules containing ubiquinone or ubiquinol. The increase in plasma levels (at 4 and $8 \mathrm{~h}$ ) was higher after intake of $100 \mathrm{mg}$ ubiquinone when compared with all other formulations ${ }^{(25)}$.

Clinical evidence. The lowest levels of myocardial CoQ10 have been observed in patients of New York Heart Association (NYHA) class IV and the highest levels in patients of NYHA class $\mathrm{I}^{(26,27)}$. The Q-SYMBIO trial $\left(\mathrm{Q}_{10}{ }^{-} \mathrm{SYMptoms,} \mathrm{BIomarker}\right.$ status [Brain-Natriuretic Peptide], and long-term Outcome [hospitalizations/mortality] $)^{(28)}$ was a multicentre, randomised placebo-controlled trial, which assessed the impact of the daily intake of CoQ10 on total mortality and not just on the surrogate endpoints. A group of 420 patients, with moderate or severe HF for a period of 2 years, were treated with $300 \mathrm{mg}$ of CoQ10 (ubiquinone) ( $n$ 202) or placebo ( $n$ 218). At the end of treatments they benefited from a significant reduction in major adverse cardiac events ( $15 \%$ of the patients in the CoQ10 group v. $26 \%$ in the placebo group; hazard ratio (HR) 0.50; $95 \%$ CI 0.32, $0 \cdot 80 ; P=0.003)$, cardiovascular mortality ( $9 v .16 \% ; P=0 \cdot 026)$, all-cause mortality (10 v. $18 \% ; P=0.018)$ and incidence of hospital stays for HF $(P=0.033)^{(28)}$. A recent meta-analysis of fourteen RCT including 2149 patients has shown that administration of CoQ10 reduces mortality risk (pooled risk ratio $=0.69$; $95 \%$ CI $0.50,0.95 ; P=0.02 ; I^{2}=0 \%$ ) and helped in improving exercise capacity (standardised mean difference (SMD) 0.62; $95 \%$ CI $0 \cdot 02,0 \cdot 30 ; P=0 \cdot 04 ; I^{2}=54 \%$ ) compared with placebo. Moreover, left ventricular (LV) ejection fraction (LVEF) also improved in CoQ10-treated subjects compared with controls (SMD 0.62; $95 \%$ CI 0.02, $\left.1 \cdot 12 ; P=0.04 ; I^{2}=75 \%\right)^{(29)}$, partially in contrast with what was reported in a previous meta-analysis including fewer studies ${ }^{(30)}$. 
Table 2. Nutraceuticals with clinical effects on heart failure (HF): level of evidence, tested dosages, effects on symptoms, effects on laboratory or instrumental parameters and effects on hard outcomes

\begin{tabular}{|c|c|c|c|c|c|}
\hline Nutraceuticals & Level $^{*}$ & $\begin{array}{l}\text { Active daily } \\
\text { doses }\end{array}$ & Effects on symptoms & Effects on laboratory or instrumental parameters & Effects on hard outcomes \\
\hline $\begin{array}{l}\text { Coenzyme } \\
\mathrm{Q}_{10}\end{array}$ & A & $100-300 \mathrm{mg}$ & $\begin{array}{l}\uparrow \text { Self-perceived } \\
\text { quality of life, } \\
\text { improvement in } \\
\text { NYHA class }\end{array}$ & $\begin{array}{l}\uparrow \mathrm{EF} \text { (if }>30 \% \text { ), } \uparrow \mathrm{LVEF}, \uparrow \mathrm{CO} \text { and } \mathrm{Cl}, \uparrow \mathrm{SV}, \\
\uparrow \mathrm{EDV}, \uparrow \text { exercise capacity, } \downarrow \text { ventricular } \\
\text { arrhythmias after surgery and need of inotropic } \\
\text { drugs (after cardiac surgery), } \\
\downarrow \mathrm{TNF}-\alpha, \mathrm{IL}-6, \text { hsCRP, } \uparrow \text { insulin sensitivity }\end{array}$ & $\begin{array}{l}\downarrow \text { MACE, total mortality and } \\
\text { incidence of hospital stays } \\
\text { for HF }\end{array}$ \\
\hline D-Ribose & B & $5 \mathrm{~g} / \mathrm{d}$ & $\begin{array}{l}\uparrow \text { Self-perceived } \\
\text { quality of life and } \\
\text { physical activity } \\
\text { performance }\end{array}$ & $\begin{array}{l}\uparrow \text { Vascular stiffness, ATP bioavailability, diastolic } \\
\text { function, ventilatory efficiency }\end{array}$ & Not investigated \\
\hline L-Carnitine & A & $1500-6000 \mathrm{mg}$ & $\begin{array}{l}\downarrow \text { Angina symptoms } \\
\quad(?)\end{array}$ & $\begin{array}{l}\uparrow \mathrm{LVEF}, \uparrow \mathrm{CO} \text { and Cl, } \uparrow \mathrm{SV}, \uparrow \mathrm{EDV}, \downarrow \mathrm{NP}, \\
\text { NT-proBNP, LVESD, LVEDD and LVESV, } \\
\downarrow \text { hs-CRP }, \downarrow \text { Lp(a), } \downarrow \text { body weight }\end{array}$ & $\begin{array}{l}\downarrow \text { Ventricular arrhythmias and } \\
\text { total mortality? (conflicting } \\
\text { data) }\end{array}$ \\
\hline L-Carnosine & B & $\begin{array}{l}500 \mathrm{mg} \\
\quad(\mathrm{L}-\text { carnosine }) \\
3-6 \mathrm{~g} \\
\quad \text { (magnesium } \\
\text { orotate) }\end{array}$ & $\begin{array}{l}\uparrow \text { Self-perceived } \\
\text { quality of life and } \\
\text { physical activity } \\
\text { performance }\end{array}$ & $\begin{array}{l}\uparrow \text { Peak } \mathrm{VO}_{2}, \mathrm{VO}_{2} \text { at anaerobic threshold and peak } \\
\text { exercise workload }\end{array}$ & $\begin{array}{l}\downarrow \text { Mortality? (few data } \\
\quad \text { available) }\end{array}$ \\
\hline$n$-3 PUFA & A & $1-4 \mathrm{~g}$ & Not investigated & $\begin{array}{l}\downarrow \text { TAG, hsCRP, TNF- } \alpha, \text { BNP, adhesion molecules, } \\
\uparrow \text { LVEF and LAEF, } \downarrow \text { blood pressure, } \uparrow \text { FMD, } \\
\downarrow \text { PWV }\end{array}$ & $\begin{array}{l}\downarrow \text { Cardiovascular mortality } \\
\text { (epidemiological data), } \\
\text { cardiac death and sudden } \\
\text { death } \\
\text { post-myocardial infarction }\end{array}$ \\
\hline Probiotics & B & $>3.5 \mathrm{CFU} / \mathrm{d}$ & $\begin{array}{l}\uparrow \text { Self-perceived } \\
\text { quality of life }\end{array}$ & $\begin{array}{l}\uparrow \text { LVEF, } \uparrow \text { LAD, } \downarrow \text { total cholesterol, LDL-cholesterol, } \\
\text { TAG, FPG, insulin levels, HOMA index, BMI, } \\
\text { waist circumference, SBP, DBP and low-grade } \\
\text { inflammation (TNF- } \alpha \text {, IL-6, hsCRP, F2- } \\
\text { isoprostane) }\end{array}$ & $\begin{array}{l}\text { Indirect correlation between } \\
\text { intestinal dysbiosis and } \\
\text { CVD }\end{array}$ \\
\hline
\end{tabular}

BNP, brain natriuretic peptide; CFU, colony-forming units; $\mathrm{Cl}$, cardiac input; $\mathrm{CO}$, cardiac output; DBP, diastolic blood pressure; EDV, end-diastolic volume; EF, ejection fraction; FMD, flow-mediated dilation; FPG, fasting plasma glucose; HOMA, homeostatic model assessment; hsCRP, high-sensitivity C-reactive protein; LAD, left atrial diameter; LAEF, left atrial emptying function; Lp(a), lipoprotein (a); LVEDD, left ventricular end diastolic diameter; LVEF, left ventricular ejection fraction; LVESD, left ventricular end systolic diameter; LVESV, left ventricular end systolic volume; MACE, major adverse cardiac events; NP, natriuretic peptide; NT-proBNP, N-terminal pro-B-type natriuretic peptide; NYHA, New York Heart Association; PWV, pulse wave velocity; SBP, systolic blood pressure; SV, stroke volume.

* See Table 1 for classification of the level of evidence.

The heterogeneity of results obtained on EF may therefore be partly explained by many factors such as the diversity of CoQ10 supplemented through different pharmaceutical forms and dosages (CoQ10 plasma concentrations are very variable and were reported in few RCT) ${ }^{(31-33)}$, diversity of HF grade of patients enrolled (NYHA I-IV), duration of treatment and co-treatment with conventional therapies. In particular, it has been suggested that blood CoQ10 concentrations should be $>2 \mu \mathrm{g} / \mathrm{ml}$ to improve $\mathrm{EF}$ in subjects with more severe $\mathrm{HF}^{(34)}$. Moreover, it seems that the CoQ10 effect on LVEF could be more relevant in patients untreated with statins and/or angiotensin-converting enzyme inhibitors $(+6.7 \%)$ compared with the subgroup of patients treated with these drugs $(+1 \cdot 2 \%)^{(35)}$. Finally, CoQ10 lowers the need for inotropic drugs and reduces the appearance of ventricular arrhythmias after surgery in the prevention of complications in patients undergoing cardiac surgery ${ }^{(36)}$ (Table 2).

Based on the available studies, CoQ10 has a high safety profile and at doses ranging from 60 to $600 \mathrm{mg} / \mathrm{d}$ does not cause clinically relevant adverse events ${ }^{(37,38)}$.

Expert opinion. Available meta-analyses support that supplementation with CoQ10 (especially with doses $\geq 200 \mathrm{mg} / \mathrm{d}$ ) can be of benefit in patients with chronic HF, in particular in early stage of HF, and might effect a reduction of major adverse cardiac events and total mortality.

\section{Hawthorn flavonoid fraction}

Hawthorn extract from Crataegus monogyna and oxyacantha is a flavonoid-rich herbal remedy with known anti-inflammatory, antioxidant, inotropic and coronary vasodilator effects ${ }^{(39)}$. The most studied dry ethanol ( $45 \%$ ) extract of hawtorn leaves with flowers is WS 1442 (drug:extract ratio 4-6.6:1) which contains 17.3-20.1\% oligomeric procyanidins and several flavonoids, including hyperoside, vitexin-rhamnoside, rutin and vitexin as well as triterpenoids and phenol carboxylic acids ${ }^{(40)}$. In vitro experiments with human myocardial tissue demonstrated a positive inotropic effect of hawthorn with a concentrationdependent increase of myocardial contractility accompanied by a transient rise in intracellular $\mathrm{Ca}^{(41)}$. The effect is probably mediated by cyclic AMP-independent inhibition of $\mathrm{Na}-\mathrm{K}$ ATPase and is accompanied by an improved energy turnover of myocytes ${ }^{(42,43)}$; however, in contrast to cardiac glycosides, hawthorn prolongs the potential action and the refractory period, thus possessing pronounced anti-arrhythmic properties which have been especially evaluated in animal models ${ }^{(44)}$. Hawthorn extract WS 1442 has also been shown to raise endothelial Ca levels by inhibition of sarcoplasmic/endoplasmic reticulum $\mathrm{Ca}^{2+}$-ATPase (SERCA) and activation of the inositol trisphosphate $\left(\mathrm{IP}_{3}\right)$ pathway, protecting against thrombininduced vascular barrier dysfunction and subsequent oedema formation $^{(45)}$. 
Table 3. Botanicals with clinical effects on heart failure (HF): level of evidence, tested dosages, effects on symptoms, effects on laboratory or instrumental parameters and effects on hard outcomes

\begin{tabular}{|c|c|c|c|c|c|}
\hline Botanicals & Level* $^{*}$ & Active daily doses & Effects on symptoms & $\begin{array}{l}\text { Effects on laboratory or } \\
\text { instrumental parameters }\end{array}$ & $\begin{array}{l}\text { Effects on hard } \\
\text { outcomes }\end{array}$ \\
\hline $\begin{array}{l}\text { Beetroot and } \\
\text { organic nitrates }\end{array}$ & B & $10-15 \mathrm{mmol}$ of $\mathrm{NO}_{3}^{-}$ & $\begin{array}{l}\uparrow \text { Self-perceived quality } \\
\text { of life }\end{array}$ & $\begin{array}{l}\uparrow \text { Exercise capacity, } \mathrm{CO}, \mathrm{VO}_{2} \max , \\
\text { submaximal aerobic endurance, } \\
\downarrow \text { blood pressure }\end{array}$ & Not investigated \\
\hline $\begin{array}{l}\text { Cacao and dark } \\
\text { chocolate }\end{array}$ & B & $\begin{array}{l}25-100 \mathrm{~g} \text { of dark chocolate } \\
\text { or } 400-1000 \mathrm{mg} \text { of cocoa } \\
\text { polyphenols }\end{array}$ & $\begin{array}{l}\uparrow \text { Self- perceived quality } \\
\text { of life }\end{array}$ & $\begin{array}{l}\uparrow \text { Vascular function (FMD, PWV), } \\
\text { LKB1, AMPK, NO bioavailability, } \\
\downarrow \text { NT-proBNP, total cholesterol, } \\
\text { LDL-cholesterol, insulin } \\
\text { resistance, HOMA index, SBP, } \\
\text { DBP and vascular inflammation } \\
\text { (hsCRP, ET-1) }\end{array}$ & $\begin{array}{l}\downarrow \text { HF hospitalisation, } \\
\text { CVD } \\
\text { (epidemiological } \\
\text { data) }\end{array}$ \\
\hline Hawthorn & A & $160-1800 \mathrm{mg}$ & $\begin{array}{l}\uparrow \text { Self-perceived quality of } \\
\text { life, symptom burden, } \\
\text { ability to enjoy and relax, } \\
\text { positive and negative } \\
\text { mood, sociability and } \\
\text { allegiance }\end{array}$ & $\begin{array}{l}\uparrow \mathrm{EF}, \uparrow \mathrm{LVEF}, \uparrow \text { exercise capacity, } \\
\text { maximal workload and exercise } \\
\text { tolerance, } \uparrow \text { maximal workload, } \\
\uparrow \text { endothelium stiffness, } \downarrow \text { lipid } \\
\text { peroxidation, pressure-heart rate } \\
\text { product }\end{array}$ & $\begin{array}{l}\downarrow \text { Sudden death } \\
\text { (patients with less } \\
\text { compromised LV } \\
\text { function, observed } \\
\text { in one trial only) }\end{array}$ \\
\hline
\end{tabular}

AMPK, AMP-activated protein kinase; CO, cardiac output; hsCRP, high-sensitivity C-reactive protein; DBP, diastolic blood pressure; EF, ejection fraction; ET-1, endothelin 1; FMD, flow-mediated dilation; HOMA, homeostatic model assessment; LKB1, liver kinase $\mathrm{B} 1$; LV, left ventricular; $\mathrm{LVEF}$, left ventricular ejection fraction; $\mathrm{NO}_{3}{ }^{-}$, inorganic nitrate; NT-proBNP, N-terminal pro-B-type natriuretic peptide; PWV, pulse wave velocity; SBP, systolic blood pressure.

* See Table 1 for classification of the level of evidence.

Clinical evidence. The Survival and Prognosis: Investigation of Crataegus Extract WS 1442 in congestive heart failure (SPICE) trial has investigated the effect of $900 \mathrm{mg} / \mathrm{d}$ of WS 1442 on mortality and hospitalisation rate in NYHA class II-III HF with reduced $\mathrm{EF}$ (HFrEF) patients, in a 24-month, randomised, placebo-controlled trial. A total of 2681 patients (WS 1442: $n 1338$; placebo: $n 1343$ ) were randomised and the primary endpoint was time till the first cardiac event. The authors showed that there was an insignificant trend towards cardiac mortality reduction with WS 1442 (11\% at month 24; HR 0.89; $95 \%$ CI $0.73,1.09 ; P=0.269)$. However, in the subgroup with LVEF $\geq 25 \%$, Ws 1442 reduced sudden cardiac death by $41 \%$ (HR $0.59 ; 95 \% \mathrm{CI} 0.37,0.94)$ at month $24 ; P=0.025$ ). Current data suggest that WS 1442 can potentially reduce the incidence of sudden cardiac death, at least in patients with less compromised LV function ${ }^{(46,47)}$.

Some meta-analyses of RCT investigated the efficacy of hawthorn extract on different HF parameters. One investigated the effects of hawthorn supplementation $(900$ and $1800 \mathrm{mg} / \mathrm{d}$ groups) on maximum workload; the results showed a statistically significant increase over placebo by a weighted mean difference (WMD) of $5.4(95 \% \mathrm{CI} 0 \cdot 7,10 \cdot 0) \mathrm{W}(P=0 \cdot 024)$. Moreover, at the end of the treatment phase, the treatment group differences compared with placebo for score change (four 'typical' HF symptoms, i.e. general capability, lassitude, early fatigability and effort dyspnoea) $v$. baseline were both significant for WS 1442 doses $900 \mathrm{mg} / \mathrm{d}(P=0.04)$ and $1800 \mathrm{mg} / \mathrm{d}(P=0.004)^{(48)}$. A Cochrane meta-analysis of RCT concluded that treatment with hawthorn compared with placebo was more beneficial for the physiological outcome of maximal workload (WMD $=5.35$ (95 \% CI $0.71,10.00) \mathrm{W} ; P<0.02 ; n$ 380), exercise tolerance $(\mathrm{WMD}=122.76(95 \% \mathrm{CI} 32.74,212.78) \mathrm{W} \times \min ; n$ 98) and the pressure-heart rate product, an index of cardiac oxygen consumption (WMD $=-19 \cdot 22(95 \% \mathrm{CI}-30 \cdot 46,-7 \cdot 98) \mathrm{mmHg} / \mathrm{min}$; $n$ 264). Furthermore, shortness of breath and fatigue were also significantly improved (WMD $=-5.47,95 \%$ CI $-8.68,-2 \cdot 26$; $n$ 239) ${ }^{(49)}$. These results were confirmed by a more recent meta-analysis of RCT that evaluated the data of $>600$ patients treated with quantified Crataegus extract or placebo; the subjects of the active group showed improvements in physiological outcome parameters, in particular in maximal workload, LVEF and pressure-heart rate product increase (PHRPI) at $50 \mathrm{~W}$ ergometric exercise. Moreover, the results on LVEF were independent from baseline data, while maximal workload and PHRPI were demonstrated to be related to baseline severity. Typical symptoms of HF patients, like reduced exercise tolerance, exertional dyspnoea, weakness, fatigue and palpitations, were improved more with active treatment and in subjects with more severe symptoms at baseline ${ }^{(50)}$ (Table 3).

The recommended daily dose of hawthorn extract is 320-900 mg (and active daily doses 160-1800 mg) to be taken in two or three doses per d. Adverse events reported were mild, transient and infrequent and in general comparable with placebo, including mild rash, headache, sweating, dizziness and gastrointestinal symptoms ${ }^{(51)}$.

Expert opinion. According to clinical evidence, Crataegus extracts have proven benefits regarding functional capacity, symptom control and health-related QoL in both HFrEF and HF with preserved EF (HFpEF). However, further studies are required, as a large proportion of the positive data has been obtained in patients not pharmacologically treated with the current standards of HF management.

\section{n-3 PUFA}

Accumulating evidence suggests that supplementation with $n-3$ PUFA could exert some positive effects in HF patients, especially during the early stages of the disease. Among the possible mechanisms of action that might be responsible for this is the possible 
role of the fatty acids EPA and DHA that have a direct action on the mitochondrial membrane, modifying its structure and function ${ }^{(52-54)}$. In particular, dietary supplementation with DHA at a clinically relevant dose increases DHA incorporation into phospholipids of the mitochondrial membrane and decreases the susceptibility of isolated cardiac mitochondria to undergo mitochondrial permeability transition induced by $\mathrm{Ca}^{2+}$ and stress ${ }^{(55)}$. DHA could in fact decrease viscosity of the membrane and a greater ease of movement of membrane proteins ${ }^{(56,57)}$.

Clinical evidence. In the meta-analysis of seven prospective epidemiological studies involving 176441 subjects with 5480 incident cases of $\mathrm{HF}$, the pooled relative risk for HF comparing the highest with the lowest category of fish intake was $0.85(95 \%$ CI $0.73,0.99 ; P=0.04)$; the corresponding value for marine $n-3$ PUFA was $0.86(95 \% \mathrm{CI} 0.74,1.00 ; P=0.05)^{(58)}$. In the large GISSI-HF trial the investigators enrolled patients with chronic HF of NYHA class II-IV, irrespective of cause and LVEF, and randomly assigned them to $1 \mathrm{~g} n$-3 PUFA daily ( $n$ 3494) or placebo ( $n$ 3481). Of the patients, $27 \%$ (955) died from any cause in the $n$-3 PUFA group and 1014 (29\%) in the placebo group (HR 0.91; $95 \%$ CI $0.833,0.998 ; P=0 \cdot 041)$. It was also reported that $57 \%$ of patients (1981) in the $n$-3 PUFA group and 2053 (59\%) in the placebo group died or were admitted to hospital for cardiovascular reasons (HR 0.92; $99 \%$ CI 0.849, 0.999; $P=0.009$ ). In absolute terms, fifty-six patients needed to be treated for a median duration of 3.9 years to avoid one death or forty-four to avoid one event like death or admission to hospital for cardiovascular reasons ${ }^{(59)}$. In the same trial, baseline LVEF increased with $n$-3 PUFA by $8.1 \%$ at 1 year, $11.1 \%$ at 2 years, and $11.5 \%$ at 3 years $v .6 .3 \%$ at 1 year, $8.2 \%$ at 2 years, and $9.9 \%$ at 3 years in the placebo group $(P=0.005)^{(60)}$. A metaanalysis of RCT also highlighted a significant reduction in cardiac death in the active group compared with control, in particular in subgroup analysis with EPA+DHA dosages $>1 \mathrm{~g} / \mathrm{d}(12 \cdot 9-29 \cdot 1 \%$ lower risks; $P<0 \cdot 05)^{(61)}$. Based on these data it seems that the effect of $n$-3 PUFA on HF seems to be dose- and time-related ${ }^{(62)}$.

A further trial enrolled $>200$ patients with ischaemic HF or dilated cardiomyopathy, NYHA class I-III on optimal medical treatment, who were divided into two groups: the first one received supplementation with $1000 \mathrm{mg}$ of $n-3$ PUFA for 14 weeks while the second group took a placebo. At the end of the 14 weeks, the results showed a reduction in end-diastolic and end-systolic LV dimensions by $2.5 \%(P=0.047)$ and $3.7 \%$ $(P=0.01)$, an improvement of LVEF by $3.6 \%(P=0.021)$ and a reduction of brain natriuretic peptide (BNP) levels by $34.6 \%$ $(P=0.001)$ compared with placebo ${ }^{(63)}$. Then, more recently, in a double-blind, placebo-controlled, cross-over trial, treatment with $2 \mathrm{~g} / \mathrm{d}$ of $n$-3 PUFA, compared with placebo, for 8 weeks in thirty-one patients with ischaemic HF was shown to improve LVEF (by $4.7 v .1 .7 \%$ ), E:E' ratio (early ventricular filling (E) to early annular mitral (E') velocities; decreased by $-9.47 v$. $-2.1 \%$ ), ST2 levels (decreased by $-4.5 v \cdot-2.4 \%$ ), flow-mediated dilation (increased by $44 v .11 \%$ ) and high-sensitivity C-reactive protein (hsCRP) levels (decreased by $-6 \cdot 1 v .4 .3 \%)(P<0.05$ for all) ${ }^{(64)}$. These results confirm those from previous investigations suggesting beneficial effects of EPA/DHA on haemodynamics,
LV indices and inflammation ${ }^{(65,66)}$. Of course, the prognostic significance of these changes has yet to be clarified (Table 2).

Based on the available evidence, the 2016 guidelines of the European Society of Cardiology suggest that PUFA supplementation may be considered in symptomatic HF patients to reduce the risk of cardiovascular hospitalisation and cardiovascular death $^{(67)}$.

n-3 PUFA are well tolerated beyond some mild gastrointestinal adverse events ${ }^{(68)}$. However, even in the recently published REDUCE-IT trial ${ }^{(69)}$ that demonstrated a statistically significant absolute risk reduction of $4.8 \%$ in its primary endpoint (cardiovascular death, non-fatal myocardial infarction, non-fatal stroke, coronary revascularisation, or unstable angina), the use of highdose $(2 \times 2$ g) highly purified EPA was associated with increased incidence of atrial fibrillation.

Expert opinion. The available evidence supports the supplementation of EPA and DHA to improve HF prognosis, especially in patients after myocardial infarction.

\section{Levo-carnitine (L-carnitine)}

L-Carnitine, a chemical analogue of choline, is a hydrophilic quaternary amine and is involved in several physiological activities such as lipid metabolism and mitochondrial defence. In particular, it plays an important role in lipid metabolism by acting as an obligatory cofactor for the oxidation of fatty acids and facilitating the transport of long-chain fatty acids from the cytosol to the mitochondrial matrix for $\beta$-oxidation ${ }^{(70)}$. In agreement with the 'energy starvation' hypothesis, suggesting that insufficient ATP supply (in addition to increased oxidation, inflammation and fibrosis) underlies the contractile dysfunction presenting in $\mathrm{HF}^{(71)}$, it seems that $\mathrm{L}$-carnitine might improve energy metabolism in cardiomyocytes and contribute to the improvement of clinical symptoms and cardiac function. Furthermore, L-carnitine exerts cardioprotective effects through the reduction of oxidative stress $^{(72)}$ and cardiac fibrosis ${ }^{(73,74)}$.

Clinical evidence. A meta-analysis of seventeen RCT enrolling $1625 \mathrm{HF}$ subjects showed a considerable improvement in overall cardiac function (evaluated in term of decreased NYHA class) (OR 3.47 (95\% CI 2.49, 4.82); P<0.01), LVEF (WMD = $4.1(95 \%$ CI $2 \cdot 34,5.93) \% ; P=0.01)$, stroke volume (WMD $=8.20(95 \% \mathrm{CI}$ $6.41,10 \cdot 01) \mathrm{ml} ; P=0.01)$, cardiac output (WMD $=0.9(95 \% \mathrm{CI}$ $0.76,1.01)$ litres $/ \mathrm{min} ; P<0.01)$ and ratio of the early (E) to late (A) ventricular filling velocities $(\mathrm{E} / \mathrm{A})(\mathrm{WMD}=0.2(95 \% \mathrm{CI}$ $0 \cdot 11,0.35) ; P<0 \cdot 01)$. In addition, treatment with L-carnitine also resulted in significant decreases in serum levels of BNP $(\mathrm{WMD}=-124.6(95 \% \mathrm{CI}-220.49,-28.71) \mathrm{pg} / \mathrm{ml} ; P=0.01)$, serum levels of N-terminal pro-B-type natriuretic peptide (NTproBNP) (WMD $=-510.4(95 \% \mathrm{CI}-785.42,-235.30) \mathrm{pg} / \mathrm{ml}$; $P<0 \cdot 01)$, LV end-systolic diameter (WMD $=-4 \cdot 1 \quad(95 \%$ CI $-6.57, \quad-1.55) \mathrm{mm} ; \quad P<0.01), \quad$ LV end-diastolic diameter $($ WMD $=-4.8(95 \% \mathrm{CI}-7.08,-2.49) \mathrm{mm} ; P<0.01)$ and $\mathrm{LV}$ end-systolic volume (WMD $=-20.2(95 \% \mathrm{CI}-35.6,-4.7) \mathrm{ml}$; $P<0 \cdot 01)$. No significant differences were reported in all-cause mortality, 6-min walk, and adverse events between L-carnitine and control groups ${ }^{(75)}$. These results were obtained with L-carnitine 
doses ranging from 1.5 to $6 \mathrm{~g} / \mathrm{d}$ and follow-up length ranging from $7 \mathrm{~d}$ to 3 years. In a further meta-analysis of ten RCT enrolling 925 patients, L-carnitine consumption was associated with significant reductions in serum CRP (-0.60 mg/l (5.71 nmol/1); $95 \%$ CI $-0.87,-0.32 \mathrm{mg} / \mathrm{l})$ and TNF- $\alpha$ concentrations $(-0.36$ (95\% CI $-0 \cdot 56,-0 \cdot 15) \mathrm{pg} / \mathrm{ml})^{(76)}$. Then, L-carnitine supplementation could have a mild but significant impact on body weight ${ }^{(77)}$ and plasma level of lipoprotein $(\mathrm{a})^{(78)}$. The prognostic importance and clinical relevance of these data still need to be clarified (Table 2).

L-Carnitine is in general well tolerated; dry mouth, rash and mild gastrointestinal problems could infrequently occur.

Expert opinion. L-Carnitine treatment might be effective for congestive HF patients as an adjuvant to conventional therapy, improving clinical symptoms and cardiac function, decreasing serum levels of BNP and NT-proBNP. Further research is required to more accurately assess the clinical relevance of L-carnitine administration for supporting HF care.

\section{Thiamine (vitamin $B_{1}$ )}

Thiamine (vitamin $B_{1}$ ) is an essential water-soluble vitamin required for cellular energy production. Thiamine pyrophosphate is the key coenzyme in the pentose phosphate pathway for transketolation of glucose-6-phosphate to ribose-5-phosphate ${ }^{(79)}$. Thiamine pyrophosphate is needed for the functioning of the pyruvate dehydrogenase complex (converting pyruvate to acetyl CoA) and $\alpha$-ketoglutarate dehydrogenase (converting $\alpha$-ketoglutarate to succinate) in the Krebs cycle, thus being essential for ATP metabolism ${ }^{(80)}$. In general, the recommended daily allowance of thiamine for adults is about $1.2 \mathrm{mg}$ for men and $1 \mathrm{mg}$ for women ${ }^{(81)}$. Deficiency of thiamine is far more common in underdeveloped and developing countries (in particular in individuals with chronic alcohol use, patients on total parenteral nutrition or who have undergone weight loss surgery) due to high incidence of poor nutritional status ${ }^{(82)}$. Direct impairment of myocardial energy production has been proposed as a possible basis for the development of the HF state seen in beriberi, the disease related to chronic dietary deficiency of thiamine ${ }^{(83)}$.

Clinical evidence. Multiple studies have shown that thiamine deficiency is more prevalent in HF patients than in the general population. In a meta-analysis of nine observational studies, the incidence of thiamine deficiency in patients with HF has been reported to be 2.5 times higher compared with that of control subjects without HF (OR 2.53, $95 \%$ CI 1·65, 3.87). The incidence of thiamine deficiency has ranged from 3 to $91 \%$ in various studies conducted in both in-patient and out-patient settings ${ }^{(84)}$. The main mechanisms for thiamine deficiency in $\mathrm{HF}$ are the reduction of thiamine intake and its poor absorption due to cardiac cachexia and splanchnic congestion; however, a main cause could be the increased urinary excretion determined by the treatment with high doses of loop diuretics ${ }^{(85)}$. In a crosssectional prospective observational analysis on thirty-two male NYHA II HF patients on prolonged diuretic therapy, sixteen patients received $300 \mathrm{mg} / \mathrm{d}$ of thiamine for $28 \mathrm{~d}$ : a $13.5 \%$ increase of LVEF was observed in thiamine recipients $(P=0.021)$ when compared with control ${ }^{(86)}$. Shimon et al. $^{(87)}$ randomised thirty hospitalised HF patients secondary to myocardial ischaemia and administered intravenous thiamine for $7 \mathrm{~d}$ or placebo in a double-blind manner followed by 6 weeks of oral thiamine at $200 \mathrm{mg} / \mathrm{d}$ in all patients. At the end of complete treatment patients experienced a $22 \%$ increase in LVEF as compared with the baseline value $(P<0 \cdot 01)$. Similar results were obtained in a small RCT carried out of nine symptomatic patients with HF with an increase in LVEF from 29.5 to $32.8 \%(P=0.024)$ after supplementation with oral thiamine at $300 \mathrm{mg} / \mathrm{d}$ for $28 \mathrm{~d}^{(88)}$. However, a recent RCT (fifty-two patients with HF and LVEF $<40 \%$ treated with $300 \mathrm{mg} / \mathrm{d}$ of thiamine or placebo for a period of $1 \mathrm{month}$ ) showed no significant difference in either systolic or diastolic echocardiographic parameters and dyspnoea between the two groups, even if patients in the thiamine group showed a significant improvement in peripheral oedema $(34.6 v .3 .8 \% ; P=0.005)^{(89)}$. In this study, however, the proportion of patients who were on furosemide was incredibly low (ten out of fifty-two). Second, spironolactone was prescribed to most (twenty-two out of twenty-six) patients in the thiamine group, which may have caused a decrease in the incidence of thiamine deficiency in this cohort of patients ${ }^{(90)}$.

In general, at dosages between 25 and $300 \mathrm{mg} / \mathrm{d}$, thiamine is confirmed to have an excellent profile of safety.

Expert opinion. Given the small sample size and inherent limitations of the available studies, long-term RCT with large samples are needed to confirm or not the positive effects of thiamine in patients with $\mathrm{HF}^{(79)}$.

\section{Cocoa and dark chocolate}

Cocoa (Theobroma cacao) is a rich source of polyphenols, generally varying from 12 to $18 \%$ of dry weight depending on variety, growing region and processing operations of the beans ${ }^{(91)}$. Among polyphenols, cocoa is particularly rich in flavonoids, in particular flavanols that are present as aglycones both in the monomeric and polymerised form. Among the monomeric flavanols the flavan-3-ols (37\% of total monomeric flavanols form), with (-)-epicatechin occurring in the largest quantities, represent $35 \%$ of the total content of phenolic compounds in cocoa beans (reaching concentrations of $16.5 \mathrm{mg} / \mathrm{g}$ dry weight in Costa Rica (acao) ${ }^{(92)}$. A rich source of cocoa polyphenols is dark chocolate, even if the treatment of cocoa beans through fermentation, roasting, drying and alkalisation processes is the cause of a significant loss of polyphenol content and of the antioxidant, metabolic and vascular effects. Therefore, the concentration of polyphenols in the cocoa bean is significantly different from that of cocoa powder and chocolate ${ }^{(93)}$. The mechanisms of action through which cocoa polyphenols could act on HF have not been yet fully clarified - the most reliable hypotheses concern the improvement of the endothelium-dependent vasodilator responses, mediated by increase in NO synthesis, suppression of endothelin-1 synthesis and reduction of NT-proBNP ${ }^{(94,95)}$.

Clinical evidence. In a prospective cohort study of 31917 Swedish men aged 45-79 years, moderate chocolate consumption was associated with a lower rate of HF hospitalisation or death: the multivariable-adjusted rate ratio was 0.88 (95\% CI $0.78,0.99$ ) for those consuming $1-3$ servings per month, 
0.83 (95\% CI $0.72,0.94$ ) for those consuming $1-2$ servings per week, 0.82 (95\% CI 0.68, 0.99) for those consuming 3-6 servings per week, and 1.10 (95\% CI 0.84, 1.45) for those consuming $\geq 1$ serving per $\mathrm{d}(P=0.001)^{(96)}$. However, a higher than moderate intake was not associated with a decreased risk. This effect may be attributable to the high energy content of commercially available chocolate, and therefore the risk to increase weight gain. However, in the large Physicians' Health Study, the association between chocolate consumption and incident $\mathrm{HF}$ was stronger in lean than in overweight/obese subjects ${ }^{(97)}$, making the interpretation of this relationship more complex, however, on the other hand supporting in a way the obesity paradox hypothesis ${ }^{(98)}$. In a recent small RCT, thirty-two patients with chronic HF, stable on guideline-directed medical therapy, were randomised to consume $50 \mathrm{~g} / \mathrm{d}$ of high-flavanol dark chocolate (HFDC; $1064 \mathrm{mg}$ of flavanols/d) or low-flavanol dark chocolate (LFDC; $88 \mathrm{mg}$ of flavanols per d) for 4 weeks and then crossed over to consume the alternative dark chocolate for a further 4 weeks. At the end of treatment with HFDC, plasma NTproBNP level was significantly reduced compared with baseline $(-44$ (SD 69) \%), and and in the follow-up (-41 (SD 77) \%) values, but also compared with LFDC (-33 (sD 72) \%) treated patients. HFDC also significantly reduced diastolic blood pressure compared with the values after LFDC $(-6 \cdot 7(\mathrm{SD} 10 \cdot 1) \mathrm{mmHg})^{(99)}$. In addition, it was recently shown that flavanol-rich chocolate also improves vascular function in patients with congestive $\mathrm{HF}^{(100)}$. Dark chocolate consumption in patients with HF was also related to an improvement in maximum work (W) $(P=0 \cdot 026)$, with no changes with placebo. In addition, a significant increase in protein levels was observed for liver kinase B1 (LKB1), adenosine monophosphate-activated protein kinase (AMPK) and PPAR- $\gamma$ coactivator 1- $\alpha$ (PGC1 $\alpha$ ) and in their active forms (phosphorylated AMPK and LKB1) as well as in citrate synthase(101). However, the prognostic significance of these changes has yet to be clarified (Table 3 ).

Cocoa is usually very well tolerated, both as functional food and as a dietary supplement (flavanols). The main risk with commercial chocolate is the increase in body weight, if consumption is not quantitatively controlled.

Expert opinion. More data are needed due to study limitations and inconsistency: the incidence of HF in epidemiological studies was often reported as a cumulative incidence in years without knowing the HF-free survival curves; although the results suggest that chocolate consumption may play a role in preventing $\mathrm{HF}$, it is still unclear how much chocolate consumption is needed to achieve this and whether the effect is linked to preventing or only delaying the development of HF. Finally, it is difficult to evaluate the risk of HF following consumption of different types of chocolate or different amounts of energy intake.

\section{L-Carnosine}

L-Carnosine ( $\beta$-alanyl-L-histidine) is a dipeptide produced primarily in the liver (from $\beta$-alanine, through carnosine synthase) and stored especially in the skeletal muscle and in the heart ${ }^{(102)}$. The rate-limiting step in the synthesis of $\mathrm{L}$-carnosine is represented by the tissue concentration of $\beta$-alanine (produced as a result of uracil hepatic metabolism), and the oral administration of $\beta$-alanine has been shown to increase the levels of $\mathrm{L}$-carnosine in the heart ${ }^{(103)}$. However, the intake of $\beta$-alanine at the active dose of $800 \mathrm{mg}$ at a single time can give 'pins and needles' paraesthesia that can last $1 \mathrm{~h}$, during the peak of plasma $\beta$-alanine ${ }^{(104)}$. L-Carnosine should exert a positive effect on HF patients through different mechanisms of action: the antioxidant and peroxyl radical-trapping ability at physiological concentrations due to its imidazole ring ${ }^{(105)}$, the prevention of hydroxylradicals production via chemical Fenton catalysis by $\mathrm{Fe}$ and $\mathrm{Cu}$ chelation $^{(106)}$, as well as the inhibition of the fragmentation and inactivation of $\mathrm{Cu}, \mathrm{Zn}$-superoxide dismutase by peroxyl radicals $^{(107)}$. The antioxidant properties of histidine derivatives have also been supposed to be responsible for the cardioprotective effect against ischaemia-reperfusion injury demonstrated in vitro ${ }^{(108)}$. Moreover, L-carnosine plays a sensitising action in cardiac contraction, probably related to an upregulation of Ca release from the sarcoplasmic reticulum ${ }^{(109)}$. This effect could lead to a procontractile action ${ }^{(110)}$, potentially useful in HF patients.

Clinical evidence. In an RCT, the effect of the oral administration of $500 \mathrm{mg}$ of L-carnosine for 6 months on QoL and exercise performance has been tested in fifty patients with stable HF and impaired LV function. Patients who received orodispersible L-carnosine experienced an improvement in 6-min walking test (6MWT) distance $(P=0.014)$ and an improvement in selfperceived QoL (assessed with a visual analogue scale score; $P=0.039)$ between baseline and follow-up. In addition, in an L-carnosine group compared with controls, at the end of 6 months of treatment the authors showed an improvement in peak $\mathrm{VO}_{2}(P<0 \cdot 0001), \mathrm{VO}_{2}$ at anaerobic threshold, 6MWT, peak exercise workload and self-perceived QoL assessed by the EuroQoL five dimensions questionnaire (EQ-5D test) and the visual analogue scale score ${ }^{(111)}$. A potential alternative to direct L-carnosine administration could be supplementation with magnesium orotate, which seems also to be clinically useful in $\mathrm{HF}^{(112)}$. The orotate is in fact a precursor of uridine, a pyrimidine nucleoside used from cardiac tissue as a source of $\beta$-alanine and subsequently of L-carnosine. A placebo-controlled study evaluating the effects following the administration of magnesium orotate ( $6 \mathrm{~g} / \mathrm{d}$ for 1 month, $3 \mathrm{~g} / \mathrm{d}$ for 11 months) in patients with severe congestive $\mathrm{HF}$, reported a survival rate of $75.7 \%$ in patients treated with magnesium orotate, compared with $51.5 \%$ survival for those treated with placebo $(P<0 \cdot 05)^{(113)}$. Orotate can be considered as a 'delayed release' form of $\beta$-alanine, which can be better tolerated than $\beta$-alanine itself, probably because the evolution of $\beta$-alanine proceeds gradually after orotate intake. Consequently, the intake of orotates or orotic acid could be a strategy for boosting the level of carnosine in the body and in the heart tissues ${ }^{(114)}$. Timed-release $\beta$-alanine preparations may also be employed to prevent the above-cited paraesthesia ${ }^{(115)}$, but this formulation has not yet been tested in HF patients (Table 2).

Carnosine is overall usually well tolerated, and no specific concerns have been raised till now as regards its use as a dietary supplement 
Expert opinion. Given the small sample size and inherent limitations of the available studies, long-term RCT with large samples are needed to confirm or not the positive effects of L-carnosine in patients with HF.

\section{Vitamin $D$}

Vitamin D (vitD; commonly referred to as $\mathrm{D}_{2}$ and $\mathrm{D}_{3}$, respectively, ergocalciferol and cholecalciferol) is a collection of fatsoluble steroids, obtained via endogenous production or from dietary intake, with the latter accounting for nearly $10-20 \%$ of our total supply ${ }^{(116)}$. Cholecalciferol is produced from its precursor 7-dehydrocholesterol, in the skin, after exposure to the UVB spectrum of sunlight. In its original state, vitamin $\mathrm{D}_{3}$ is inactive and requires two separate hydroxylation reactions (the first in the liver and the second in the kidney) to become biologically active (calcitriol). Calcitriol exerts its effects via the vitD receptor and it is responsible for the regulation of gene transcription and protein synthesis, with the known effect on Ca homeostasis and bone metabolism ${ }^{(117)}$. Nevertheless, in recent years, the biological influence of vitD has been significantly expanded beyond just $\mathrm{Ca}$ regulation. VitD receptor has been isolated from a number of different tissues not traditionally involved in $\mathrm{Ca}$ homeostasis, such as the myocardium ${ }^{(118)}$ and fibroblasts ${ }^{(119)}$. Despite emerging evidence supporting a pathophysiological relationship between vitD and $\mathrm{HF}$, the exact mechanism by which vitD deficiency leads to poor clinical outcome has not been clearly established. One of the hypotheses is the relationship between the heart and kidney: these two organs are interrelated and the impairment of one system can induce pathological processes within the other, thus accelerating the progressive failure of both. This interrelationship serves as the pathophysiological basis of the clinical entity called cardiorenal syndrome ${ }^{(120)}$. The progression of cardiorenal syndrome involves the renin-angiotensin-aldosterone system and sympathetic nervous system overactivation, as well as systemic inflammation, which could alter electrolyte and fluid regulation, cause endothelial dysfunction, and both LV remodelling and fibrosis along with renal fibrosis and failure ${ }^{(121,122)}$. These changes set up a vicious cycle, resulting in further systemic dysfunction until organ failure occurs. VitD is particularly low in individuals with cardiorenal syndrome, probably due to reduced $\alpha$-1-hydroxylase activity and the depletion of vitDbinding proteins secondary to proteinuria ${ }^{(123)}$. Similarly, with those HF patients, they are also associated with increased cardiovascular events ${ }^{(124)}$. In a study of patients with diabetic nephropathy, paricalcitol supplementation significantly reduces proteinuria $^{(125)}$, a known predictor of cardiovascular events ${ }^{(126,127)}$. VitD deficiency in HF patients could also be linked to an overactivation of the renin-angiotensin-aldosterone system $^{(128)}$, and to increased production and release of inflammatory cytokines, which can have a direct negative effect on the myocardium or indirectly affect other vital organs, causing myocardial apoptosis, hypertrophy, fibrosis, LV remodelling, negative ionotropic effects $^{(129-131)}$, increased renal fibrosis and renal failure ${ }^{(132,133)}$. In vitro studies suggest that vitD suppresses proinflammatory cytokines such as TNF- $\alpha$ and IL- 6 and up-regulates anti-inflammatory cytokines such as IL-10 ${ }^{(134)}$, while the lack of vitD is associated with increased myocardial matrix metalloproteinase expression, increased collagen deposition and fibrosis ${ }^{(135,136)}$.

Clinical evidence. Many observational studies have suggested a possible relationship between vitD status and prevalence, incidence and severity of $\mathrm{HF}^{(137,138)}$. In a North-American study carried out on 8351 participants, individuals in the lowest tertile were approximately twice as likely to have HF compared with those in the highest tertile (OR 2.1; $95 \%$ CI $1 \cdot 2,3 \cdot 6)^{(139)}$. Similar results were confirmed in the larger Intermountain Heart Collaboration Study in a cohort of 41504 patients $^{(140)}$. In the German Ludwigshafen Risk and Cardiovascular Health Study with 3299 patients referred for coronary angiography, a significant negative correlation was observed between serum 25-hydroxyvitamin D (25(OH)D) and NT-proBNP concentration $(r-0 \cdot 2 ; P<0 \cdot 001)$ and mean serum 25(OH)D concentration was positively correlated with LVEF and negatively correlated with NYHA functional class ${ }^{(141)}$. 25(OH)D levels were negatively associated with $\mathrm{N}$-terminal pro-A-type natriuretic peptide in NYHA II-IV patients ${ }^{(142)}$. In addition, individuals with a serum $25(\mathrm{OH}) \mathrm{D}$ concentration $<10 \cdot 0 \mathrm{ng} / \mathrm{ml}(<25 \cdot 0 \mathrm{nmol} / \mathrm{l})$ had significantly larger LV volumes compared with those with a serum $25(\mathrm{OH}) \mathrm{D}$ concentration $>10 \cdot 0 \mathrm{ng} / \mathrm{ml}(>25.0 \mathrm{nmol} / \mathrm{l})$, and mean fractional shortening was significantly lower in those with a serum 25(OH)D concentration $<10.0 \mathrm{ng} / \mathrm{ml}(<25.0 \mathrm{nmol} / \mathrm{l})$ $(31.6 v .37 \cdot 1 \% ; P<0 \cdot 05)^{(143)}$. In general, mean serum $25(\mathrm{OH}) \mathrm{D}$ concentration is usually lower in patients with HF compared with controls $^{(144-146)}$. VitD status may be related to physical functioning in HF: there seems to be a negative correlation between cardiopulmonary stress test performance and serum $25(\mathrm{OH}) \mathrm{D}$ concentration $\leq 9.0 \mathrm{ng} / \mathrm{ml}(\leq 22.5 \mathrm{nmol} / \mathrm{l})$ compared with those with levels $\geq 10 \cdot 0 \mathrm{ng} / \mathrm{ml}(\geq 25 \cdot 0 \mathrm{nmol} / \mathrm{l})^{(147)}$. A significant positive correlation between serum 25(OH)D concentration, 6MWT distance $(r 0.4 ; P<0 \cdot 05)^{(148)}$ and maximal oxygen uptake during cardiopulmonary stress testing was observed in $\mathrm{HF}$ patients ${ }^{(149)}$. VitD status may also be related to prognosis in HF: in the above-cited Ludwigshafen Risk and Cardiovascular Health Study (patients were followed-up for a median of 7.7 years), individuals with severe vitD deficiency (serum $25(\mathrm{OH}) \mathrm{D}$ concentration $<10.0 \mathrm{ng} / \mathrm{ml}(<25.0 \mathrm{nmol} / \mathrm{l}))$ were approximately four times more likely to die from $\mathrm{HF}$ than those with a serum $25(\mathrm{OH}) \mathrm{D}$ concentration in the optimal range $(>30.0 \mathrm{ng} / \mathrm{ml}(>75.0 \mathrm{nmol} / \mathrm{l}))(\mathrm{HR} 4 \cdot 1 ; 95 \% \mathrm{CI} 1 \cdot 8,9 \cdot 6)$. Even patients waiting for heart transplantation in the highest $25(\mathrm{OH}) \mathrm{D}$ tertile were approximately twice as likely to survive in comparison with those in the lowest tertile (HR 0.4; $95 \%$ CI $0 \cdot 3,0 \cdot 5)^{(150)}$.

In an RCT, 123 patients with HF treated with vitD (2000 IU/d) $+\mathrm{Ca}(500 \mathrm{mg} / \mathrm{d})$ for 9 months experienced significantly increased IL-10 and reduction of TNF- $\alpha$ compared with placebo (administered with at $\mathrm{Ca} 500 \mathrm{mg} / \mathrm{d}$ only) ${ }^{(151)}$. In paediatric HF patients, daily vitD supplementation with 1000 IU of cholecalciferol resulted in significant improvement in LV end diastolic and systolic diameters, LVEF and myocardial performance index, together with reductions in inflammatory cytokines ${ }^{(152)}$. VitD supplementation (between 800 and $1000 \mathrm{IU} / \mathrm{d}$ ) in HF patients with low 25(OH)D levels was associated with a significant reduction in mortality, independent of the baseline $25(\mathrm{OH}) \mathrm{D}$ levels ${ }^{(153)}$. 
However, supplementation with a very high dosage (100 000 IU/ week) was not associated with improvement of 6MWT distance after 20 weeks of treatment, despite a significant reduction in BNP levels ${ }^{(154)}$, probably because in this study the obtained serum 25(OH)D levels were not optimised.

In general, vitD supplementation is believed to be safe because it rarely raises serum vitD levels to the toxic range even after repeated intravenous ingestion of extremely high doses of synthetic vitD. However, prolonged consumption of vitD supplementation may induce hypercalcaemia, hypercalciuria and hyperphosphataemia, which are considered the initial signs of vitD intoxication $^{(155)}$. Use of thiazide diuretics in combination with $\mathrm{Ca}$ and vitD supplements may cause hypercalcaemia in the elderly or those with compromised renal function or hyperparathyroidism ${ }^{(156)}$.

Expert opinion. It seems that vitD might be useful in the supporting HF therapy and improve prognosis. However, largescale randomised, multicentre clinical trials are still needed before routine vitD supplementation can be recommended as part of clinical care of HF patients. VitD should be supplemented in vitD-deficient subjects, and especially in those with heart diseases.

\section{Magnesium}

Mg plays a role in many enzymic processes, contributing to stable cardiovascular haemodynamics and electrophysiological functioning: it is an important component in mitochondrial function, modulating cellular $\mathrm{K}$ permeability and affecting $\mathrm{Ca}$ uptake and its distribution $^{(157,158)}$. In respect of other electrolyte alterations, the pathophysiology of hypomagnesaemia (serum $\mathrm{Mg}<1.5 \mathrm{mg} / \mathrm{dl}(0.62 \mathrm{mmol} / \mathrm{l}))$ remains less studied even if is not infrequently observed in HF patients: the prevalence is about $7 \%$ of well-compensated ambulatory subjects to $52 \%$ in more advanced HF patients who are aggressively treated with diuretics ${ }^{(159)}$. To date, it is rather clear that the effective correction of $\mathrm{Mg}$ disturbances is favourable in HF subjects, in particular in preventing potentially life-threatining arrhythmias ${ }^{(160)}$. In HF, $\mathrm{Mg}$ depletion stems from several factors such as reduced dietary intake, altered distribution of the ion, renal losses, oedematous states (involving the intestinal mucosa that might interfere with the absorption of microelements), respiratory alkalosis and excessive catecholamine release ${ }^{(161)}$. Moreover, diuretics produce most of renal $\mathrm{Mg}$ loss, especially in the volume-expanded setting of $\mathrm{HF}$ and in associated hyperaldosteronism and it has been demonstrated that $\mathrm{K}$ depletion inhibits the renal reabsorption of $\mathrm{Mg}$, leading to hypermagnesiuria and hypomagnesaemia ${ }^{(162)}$. Hypomagnesaemia seems to have vasoconstrictor properties due to the inhibition of PG-induced relaxation and to the enhancement of the activity of the vasoconstrictor neurohormones through alterations in $\mathrm{Ca}$ uptake. In HF patients, the presence of adequate total-body $\mathrm{Mg}$ stores has been associated with a reduction in the risk of arrhythmias, digitalis toxicity and haemodynamic abnormalities ${ }^{(160)}$.

Clinical evidence. Most of the evidence supporting Mg supplementation in HF patients comes from observational data. Small
RCT carried out in HF patients have underlined that Mg supplementation improves $\operatorname{LVEF}^{(163)}$ and heart rate variability ${ }^{(164)}$. On the other hand, observational studies have linked low serum Mg to more adverse CVD risk factor profiles ${ }^{(165-167)}$ and greater risk of CVD events ${ }^{(168)}$. In the Atherosclerosis Risk in Communities (ARIC) study, participants in the lowest quintile of $\mathrm{Mg}$ levels were at 2.5 times greater risk of incident HF after adjustment for demographic factors, and with additional adjustment for behaviours and CVD risk factors ${ }^{(169)}$. HF worsening secondary to very severe hypomagnesaemia has been described, and, in some cases, $\mathrm{Mg}$ supplementation determines the reversal of $\mathrm{HF}^{(170)}$. Overall, among patients with $\mathrm{HF}$, low serum $\mathrm{Mg}$ has been associated with increased all-cause mortality ${ }^{(171)}$. The British Heart Regional Study (a prospective study of 3523 men aged 60-79 years with no prevalent HF or myocardial infarction followed up for a mean period of 15 years) showed that serum $\mathrm{Mg}$ was inversely related to risk of incident HF (after adjustment for conventional CVD risk factors and incident myocardial infarction). In addition, the benefit of high serum $\mathrm{Mg}$ on HF risk was most evident in men with electrocardiogram evidence of ischaemia (HR 0.29; $95 \%$ CI 0.13, 0.68; $P<0.05)^{(172)}$. However, a meta-analysis of seven eligible prospective studies did not observe a clear correlation between hypomagnesaemia and HF, if not in elderly HF patients with reduced $\operatorname{LVEF}^{(173)}$. However, in the Jackson Heart Study cohort, $\mathrm{Mg}$ intake $<2.3 \mathrm{mg} / \mathrm{kg}$ was related to increased risk of subsequent HF hospitalisations ${ }^{(174)}$, and, in a more recent study, Mg deficiency independently predicted poor QoL and earlier cardiac event-free survival in HF patients ${ }^{(175)}$. Finally, a recent meta-analysis showed a clear and significant link between $\mathrm{Mg}$ supplementation and reduction of inflammation ${ }^{(176)}$

Low serum Mg seems to be associated with an increased risk of incident atrial fibrillation ${ }^{(177,178)}$ that is closely related with $\mathrm{HF}^{(179,180)}$. In particular, many studies have highlighted the correlation between serum $\mathrm{Mg}$ deficiency and atrial fibrillation risk after cardiac surgery: in that setting, $\mathrm{Mg}$ is sometimes used prophylactically to prevent atrial fibrillation events ${ }^{(181-183)}$. In addition, $\mathrm{Mg}$ is believed to be linked to CVD risk through a broad range of physiological roles; low serum concentrations have been associated with impaired glucose homeostasis and insulin action, elevated blood pressure, chronic inflammation, impaired vasomotor tone and peripheral blood flow, and electrocardiogram abnormalities ${ }^{(184)}$. A recent meta-analysis of forty prospective cohort studies totalling more than 1 million participants has shown that increasing dietary $\mathrm{Mg}$ intake is associated with a reduced risk of stroke, $\mathrm{HF}$, diabetes, and all-cause mortality, but not CHD or total CVD ${ }^{(185)}$.

Mild diarrhoea, stomach upset, nausea and heartbeat are the most common dose-dependent side effects after the use of Mg. $\mathrm{Mg}$ toxicity rarely occurs except in patients with renal dysfunction: severe kidney failure and heart block are the two relative contraindications $^{(186)}$.

Expert opinion. The available data suggest that we should avoid hypomangnesaemia in patients with HF. However, the prognostic significance of serum $\mathrm{Mg}$ concentration in HF patients as well as clinical relevance of its supplementation still needs to be investigated. Additional work is needed to elucidate whether 
the association between HF and Mg deficiency is causal and to clarify the specific mechanisms that underlie the association.

\section{Beetroot and inorganic nitrates}

$\mathrm{NO}$ is not only an endothelium-derived relaxing factor, but is also a key cellular signalling molecule with pleiotropic effects in many tissues. For example, in skeletal muscle NO helps to modulate contractile function, through the nitrosation or S-nitrosylation of various proteins ${ }^{(187)}$. In addition, during concentric activity, NO significantly increases the rate of force development, maximal shortening velocity, and maximal power of both single muscle fibres and isolated muscles via the activation of the classic NO soluble guanyl cyclase-cycle GMP (NO-sGCcGMP) pathway ${ }^{(188)}$. In failing cardiac muscle, however, the increased production of reactive oxygen species leads to more rapid destruction of $\mathrm{NO}$, and hence reduced NO-sGC-cGMP signalling, which in turn is thought to contribute to reduced contractility in $\mathrm{HF}^{(189)}$. It is possible therefore that $\mathrm{NO}$ bioavailability is diminished in the skeletal muscles of patients with HF, thus contributing to their reduced muscle function ${ }^{(188)}$. It is also well established that HF leads to endothelial dysfunction in various tissues, including skeletal muscle, as a result of reduced NO production via endothelial NO synthase ${ }^{(190)}$. Emerging evidence suggests that supplementation of dietary inorganic nitrate $\left(\mathrm{NO}_{3}^{-}\right)$has beneficial effects on vascular health, blood pressure, exercise capacity and oxygen metabolism though targeted NO production ${ }^{(191)}$. After ingestion, $\mathrm{NO}_{3}{ }^{-}$is reduced to bioactive $\mathrm{NO}_{2}{ }^{-}$by bacteria found in the oral cavity. $\mathrm{NO}_{2}{ }^{-}$is then taken up by the plasma from the digestive system and can be converted to NO, particularly under hypoxic or acidic conditions ${ }^{(192)}$, which can occur in $\mathrm{HF}$ and during exercise. Inorganic nitrates slowly produce NO and determine more mild but sustained vasodilation while organic nitrates rapidly release relatively large amounts of $\mathrm{NO}^{(193,194)}$, with potentially adverse effects on myocardial functionality in HF patients ${ }^{(195)}$. Compared with placebo, either a single, acute dose or 1 week of daily dosing of beetroot juice (BRJ) significantly increased plasma $\mathrm{NO}_{2}{ }^{-}$similarly, by 138 and $129 \%$ compared with placebo, respectively ${ }^{(196)}$.

Clinical evidence. In some RCT, BRJ has been shown to increase time to exhaustion during high-intensity exercise and to reduce oxygen consumption $\left(\mathrm{VO}_{2}\right)$ during submaximal exercise (i.e. reduce oxygen cost at a given submaximal work rate $)^{(197-199)}$. These results were obtained not only in healthy individuals but also in HF patients. In these latter subjects, a single dose of BRJ (12.9 $\mathrm{mmol} \mathrm{NO}_{3}^{-}$) was shown to increase total work performed and cardiac output compared with placebo, while decreasing systemic vascular resistance and reducing aortic augmentation index during a maximal exercise test ${ }^{(200)}$. In healthy older adults, BRJ supplementation has been shown to improve $\mathrm{VO}_{2}$ kinetics ${ }^{(201)}$. Daily supplementation with BRJ containing $11.2 \mathrm{mmol}$ of $\mathrm{NO}_{3}{ }^{-}$increased $\mathrm{NO}$ production and muscle function with an improvement of physical exercise in HF patients ${ }^{(202)}$. In another RCT, 1 week of daily dosing with BRJ containing $6 \cdot 1$ mmol $\mathrm{NO}_{3}{ }^{-}$significantly improved submaximal aerobic endurance $(+24 \% ; P=0.02$ compared with placebo) and blood pressure $(P<0 \cdot 001)$ in elderly patients with $\mathrm{HFpEF}^{(203)}$.
Acute dietary $\mathrm{NO}_{3}{ }^{-}$intake was also related to an increase of $\mathrm{VO}_{2}$ peak and improved physical performances in HFrEF patients $^{(204)}$. BRJ may have positive effects combined with an aerobic exercise training regimen in HFrEF patients, even if data are still limited and inconsistent ${ }^{(205)}$. BRJ has also been shown to improve exercise capacity and oxygen metabolism in older patients with peripheral arterial disease ${ }^{(206)}$. Data on EF and hard outcomes are still lacking; however, a meta-analysis of RCT including 1248 patients showed that BRJ is associated with significant reductions in blood pressure (systolic blood pressure: -3.55 (95\% CI $-4.5,-2.5) \mathrm{mmHg}$; diastolic blood pressure: $-1.3(95 \% \mathrm{CI}-1.9$ to $-0 \cdot 7) \mathrm{mmHg}$ ) compared with controls, thus reducing the afterload. The mean difference of SBP was larger between BRJ-supplemented and control groups in the longer than in the shorter $(\geq 14$ compared with $<14 \mathrm{~d}$ ) study durations $(-5 \cdot 1$ compared with $-2.7 \mathrm{mmHg}$ ) and the highest compared with the lowest (500 compared with 70 and $140 \mathrm{ml} / \mathrm{d}$ ) doses of BRJ ( -4.8 compared with $-2.4 \mathrm{mmHg})^{(207)}$. Long-term effects on EF and hard outcomes are lacking (Table 3).

In general, BRJ supplementation seems to be tolerable and safe after short-term administration. However, most available clinical trials are short term $(<30 \mathrm{~d})$. Long-term data are lacking.

Expert opinion. Taking into account relatively limited data, mainly short term, on the possible administration of beetroot and $\mathrm{NO}_{3}{ }^{-}$in HF patients it is difficult to draw any recommendations of its usefulness. Therefore, large-scale randomised, multicentre clinical trials are still needed.

\section{D-Ribose}

D-Ribose is a pentose carbohydrate that plays many physiological roles. Myocardial ATP levels are important to maintain cell integrity and function: ATP has a key role in the interaction between $\mathrm{Ca}$ and the sarcoplasmic reticulum (important for ventricular relaxation) ${ }^{(208)}$. In fact, deficient ATP levels are found in IHD and $\mathrm{HF}^{(209)}$ and can be responsible for diastolic dysfunction and a non-compliant ventricle. Exogenous supplementation with D-ribose enhances the regeneration of ATP levels by bypassing rate-limiting, slow enzymic steps in glycolysis through the pentose phosphate pathway ${ }^{(210,211)}$. In an experimental model of ischaemic injury, supplementation with D-ribose might quickly increase immediately the levels of ATP, improving diastolic dysfunction and substantially shortening the lengthy time recovery that normally occurs following ischaemia ${ }^{(212)}$.

Clinical evidence. Preliminary results in HF patients confirm what was observed in experimental models: in a prospective feasibility study, D-ribose improved diastolic dysfunction, selfperceived QoL and physical function ${ }^{(213)}$, while in a pilot trial D-ribose improved ventilatory efficiency ${ }^{(214)}$. In an RCT, the effects of the administration of $5 \mathrm{~g}$ daily of D-ribose for 6 weeks were evaluated in patients with NYHA II-IV. At the end of the treatment, the results showed an improvement of tissue Doppler velocity (E') (in $75 \%$ of the patients) what was also maintained in the follow-up visit at 9 weeks (i.e. 3 weeks after the treatment with D-ribose was stopped). In addition, half of the patients achieved an improvement in their ratio of $\mathrm{E}: \mathrm{E}^{(215)}$. 
At least part of these effects could be related to the anti-ischaemic properties of D-ribose: supplementation with this pentose carbohydrate enabled patients with stable coronary artery disease to exercise longer without developing angina or electrocardiographic changes ${ }^{(216)}$. Moreover, improvement in heamodynamic parameters has also been recorded perioperatively, in patients undergoing off-pump coronary artery bypass ${ }^{(217)}$ and in individuals following aortic valve replacement ${ }^{(218)}$ (Table 2).

Side effects were negligible.

Expert opinion. D-Ribose might offer an energetic benefit in patients with ischaemic CVD, including HF (particularly; with the improvement of diastolic dysfunction). Even if the preliminary data are encouraging, clinical studies conducted to date are still few, small and relatively short.

\section{Probiotics}

Recent clinical and preclinical studies underline the key role of intestinal microbiota in cardiovascular health and in HF prognosis in particular ${ }^{(219)}$. Intestinal eubiosis is important in regulation of the function of the intestinal barrier, together with mucosal immunity, $\mathrm{Na}$ and water homeostasis and the functionality of tight junctions. In particular, subjects with HF manifest gastrointestinal disorders of absorption, motility, tissue perfusion and oedema, which determine alterations of the intestinal bacterial flora that in long term are responsible for an increase in translocation of endotoxins in the blood, an increase in preload and afterload and an aggravation of the clinical picture ${ }^{(220,221)}$.

Clinical evidence. A strong correlation appears to exist between HF severity and the severity of intestinal dysbiosis, measured through the serum levels of trimethylamine $\mathrm{N}$-oxide (TMAO), an amine produced by the metabolism of choline and phosphatidylcholine from intestinal microbiota (especially from opportunistic/pathogenic micro-organisms) ${ }^{(222)}$. It is hypothesised that vascular remodelling and progressive coronary atherogenesis may occur in the context of high levels of TMAO ${ }^{(223)}$. The aetiopathogenetic mechanism is not clear yet; however, it is evident that there is a direct proportionality between the blood levels of TMAO and an increase in intestinal oedema, inflammatory metabolites and cardiac and vascular remodelling ${ }^{(224)}$. In a recent prospective study, the potential pathophysiological role of intestinal microbiota in HF and its relationship to mortality from all causes was examined; in particular, the authors studied (in 720 subjects and for a duration of 5 years of follow-up) the role of TMAO, measured through fasting blood samples. The study found that the highest TMAO levels were reported in patients with HF (mean TMAO levels: $5 \cdot 0 \mu \mathrm{M}$ ) compared with healthy subjects (mean TMAO levels: $3.5 \mu \mathrm{m} ; P<0.001$ ), with a risk of mortality increased by 3.4 times ${ }^{(225)}$. Finally, it has been shown that elevated TMAO levels modify lipid metabolism through changes in the functionality of reverse cholesterol transport, sterol metabolism and modification of the quality and quantity of bile acids ${ }^{(226,227)}$. An RCT conducted in patients with NYHA class II or III and LVEF $<50 \%$, treated for 3 months with a preparation containing $1000 \mathrm{mg} / \mathrm{d}$ of probiotics (Saccharomyces boulardii), evaluated the efficacy of this supplementation on haemodynamic parameters. At the end of 3 months of treatment, the group treated with probiotics benefited from a significant reduction in uric acid levels $(-1.08 \mathrm{mg} / \mathrm{dl}(-64.24 \mu \mathrm{mol} / \mathrm{l}), P=0.014 v$. placebo: $-0.01 \mathrm{mg} / \mathrm{dl}(-0.59 \mu \mathrm{mol} / \mathrm{l}), \quad P=0.930)$, total cholesterol $(-7.63 \mathrm{mg} / \mathrm{dl}(-0.20 \mathrm{mmol} / \mathrm{l}), P=0.010 v$. placebo: $-2.02 \mathrm{mg} / \mathrm{dl}$ $(-0.05 \mathrm{mmol} / \mathrm{l}), P=0.603)$, hsCRP $(-0.23 \mathrm{mg} / \mathrm{dl}(-2.3 \mathrm{mg} / \mathrm{l})$, $P=0.116 v$. placebo: $+0.44 \mathrm{mg} / \mathrm{dl}(+4.4 \mathrm{mg} / \mathrm{l}), P=0.011)$, improvement in LVEF $(+6.6 \%, P=0.005 v$. placebo: $+4.2 \%$, $P=0.173)$ and of the left atrial diameter $(-0.29 \mathrm{~cm}, P=0.044 \mathrm{v}$. placebo: $+0 \cdot 2 \mathrm{~cm}, P=0 \cdot 079)^{(228)}$. Preclinical data have shown results comparable with Lactobacillus rhamnosus ${ }^{(229)}$. A recent study by Maier et al. ${ }^{(230)}$ showed that about one-quarter of nonantibiotic drugs are able to inhibit the growth of at least one main strain of intestinal bacterial flora. Among the cardiovascular drugs, the most interesting ones appear to be $\mathrm{Ca}$ antagonists and numerous anti-arrhythmics. Therefore, the microbiome dysbiosis-drug interaction in HF patients is particularly evident compared with the general population and so greater attention should be paid to possible alterations and new aetiopathogenetic mechanisms caused to the microbiome ${ }^{(225)}$. The ongoing GutHeart trial has randomised 150 patients with stable HF and a $\mathrm{LVEF}<40 \%$ to receive the antibiotic rifaximin, the probiotic yeast S. boulardii (ATCC 74012) or no treatment in a 1:1:1 fashion. The primary endpoint is EF at 3 months. The outcomes of the trial will shed some light into the possible therapeutic avenues in the future targeting the gut microbiome (Table 2).

In general, supplementation with probiotics in cardiovascular prevention has proved to be safe and free of any relevant side effects.

Expert opinion. Some probiotic strains (in particular lactobacilli, bifidobacterial, in addition to $S$. boulardii) could be applied as an adjuvant to conventional therapy in HF treatment. Further longterm clinical trials (including the results from the Gut-Heart trial) are necessary to investigate the effects of probiotic supplementation on cardiovascular outcomes.

Iron

Fe deficiency is defined in the general population as serum ferritin $<30 \mathrm{ng} / \mathrm{ml}$ and transferrin saturation below $20 \%$; however, different cut-off values are used in the presence of inflammatory co-morbidities (ferritin is an acute-phase protein that increases during inflammation) such as inflammatory bowel disease (ferritin $<100 \mathrm{ng} / \mathrm{ml}$ ), chronic kidney disease (ferritin $<500 \mathrm{ng} / \mathrm{ml}$ plus transferrin $<30 \%$ ) and chronic kidney disease (transferrin $<100 \mathrm{ng} / \mathrm{ml}$ or $<100-299 \mathrm{ng} / \mathrm{ml}$ plus transferrin $<20 \%$ ) to diagnose Fe deficiency ${ }^{(231)}$. The burdening prevalence of this co-morbid condition is illustrated by the findings of such deficit in 30-50\% in chronic stable disease ${ }^{(232,233)}$ and $-80 \%$ in acute $\mathrm{HF}^{(234,235)}$. Furthermore, Fe deficiency independently predicts more severe symptomatic burden, higher morbidity, as noted by markedly increased hospitalisations and readmission rates, and mortality ${ }^{(236)}$, which underscores its importance in HF. Fe deficiency in HF patients (as in the general population) may be due to either reduced $\mathrm{Fe}$ intake, increased $\mathrm{Fe}$ body losses and/or impaired $\mathrm{Fe}$ absorption $^{(237)}$. Briefly, anaemia is often the result of compromised $\mathrm{Fe}$ stores and/or impaired transportation. In addition to $\mathrm{Fe}$ 
deficiency, multiple other factors can contribute to anaemia, including inflammation, renal dysfunction and haemodilution ${ }^{(238)}$.

Clinical evidence. A small study noted that Fe intake was markedly decreased in HF patients, aggravating with increased disease severity ${ }^{(239)}$. However, the Iron Repletion effects ON Oxygen UpTake in Heart Failure (IRONOUT HF) multicentre double-blind RCT, that included 255 patients with symptomatic Fe deficiency and HFrEF treated for 16 weeks with oral intake of $150 \mathrm{mg}$ twice daily of Fe polysaccharide or placebo, found no significant differences between groups regarding natriuretic peptide levels, symptomatic score, 6MWT or peak $\mathrm{VO}_{2}$. This RCT also does not support a role for oral Fe in Fe deficiency in HFrEF patients ${ }^{(240)}$. Today, no trial has formally tested the role of oral Fe in patients with $\mathrm{HF}$ and mid-range $\mathrm{EF}$ (HFmrEF) or HF with HFpEF. According to 2016 European Society of Cardiology guidelines for the diagnosis and treatment of acute and chronic $\mathrm{HF}$, intravenous ferric carboxymaltose should be considered in symptomatic patients with HFrEF and Fe deficiency (serum ferritin $<100 \mu \mathrm{g} / \mathrm{l}$, or ferritin between 100 and $299 \mu \mathrm{g} / \mathrm{l}$ and transferrin saturation $<20 \%$ ) in order to alleviate HF symptoms, and improve exercise capacity and QoL ${ }^{(61)}$. While intravenous Fe administration in stable symptomatic (NYHA II-III) chronic HFrEF is supported by several clinical trials, no convincing evidence is available regarding oral Fe supplementation ${ }^{(241)}$.

Whether low bioavailability due to intestinal wall oedema, adverse events and subsequent low rates of adherence, and polypharmacy hinder the benefits of oral Fe in these subpopulations is currently unknown. Moreover, oral Fe supplementation is often associated with multiple unwanted events, particularly gastrointestinal adverse effects ${ }^{(242)}$, which outweigh the null benefit of such dietary supplement in HFrEF.

Expert opinion. The authors of this Position Paper support the recent European Society of Cardiology guidelines as for potential Fe supplementation in HFrEF patients. Further studies are necessary as for potential benefits of oral supplementation and in other types of HF (HFpEF and HFmrEF).

\section{Vitamin C}

Ascorbic acid (vitamin C) protects against oxidative stress by reducing levels of free oxygen radicals and inhibiting LDL oxidation and oxidative cell damage ${ }^{(243)}$. In addition, it is responsible to improve arterial stiffness and immune function, and to reduce inflammatory markers responsible for systemic inflammation ${ }^{(244)}$.

Clinical evidence. Vitamin C is contained mostly in fruits and vegetables and some ${ }^{(245,246)}$, but not all the studies ${ }^{(247)}$ have underlined that a diet high in fruit and vegetables, rich in antioxidants, is associated with a reduced risk of HF. Moreover, only a few studies have examined the association between plasma vitamin $\mathrm{C}$ levels and incidence of $\mathrm{HF}$, suggesting a positive correlation between elevated plasma vitamin $\mathrm{C}$ levels and reduction of the HF risk ${ }^{(248)}$. In a prospective study including 3919 men aged 60-79 years with no $\mathrm{HF}$ at baseline and followed up for a mean period of 11 years, a higher plasma vitamin $\mathrm{C}$ level was associated with a significantly lower risk of incident $\mathrm{HF}$ in both men with and without previous myocardial infarction after adjustment for lifestyle characteristics, diabetes mellitus, blood lipids, blood pressure and heart rate (HR 0.81 (95\% CI 0.70, $0.93)$ and $0.75(95 \%$ CI $0.59,0.97)$ for 1 SD increase in log vitamin C, respectively $)^{(249)}$. In a recent study, $200 \mathrm{HF}$ patients completed a $3 \mathrm{~d}$ food diary to determine vitamin C intake: $39 \%$ of patients had vitamin $\mathrm{C}$ deficiency that was associated with an hsCRP level higher than $3 \mathrm{mg} / \mathrm{l}$ in the hierarchical logistic regression (OR $2.40 ; 95 \%$ CI $1.13,5 \cdot 10 ; P=0.023)$. In addition, both vitamin $\mathrm{C}$ deficiency and hsCRP level higher than $3 \mathrm{mg} / \mathrm{l}$ predicted shorter cardiac event-free survival in hierarchical Cox regression and produced a $2 \cdot 3$-fold higher risk for cardiac events $(P=0 \cdot 002)$ in moderation analysis. Higher levels of hsCRP predicted shorter cardiac event-free survival only in patients with vitamin $\mathrm{C}$ deficiency $(P=0.027)$, but not in those with vitamin $C$ adequacy ${ }^{(250)}$. In addition, a meta-analysis of forty-four RCT showed a significant positive effect of vitamin $\mathrm{C}$ on endothelial function (SMD $0.50,95 \%$ CI $0.34,0 \cdot 66 ; P<0.001)$, in particular in the HF subgroup (SMD 0.48; $95 \%$ CI 0.08, 0.88; $P<0.02)^{(251)}$. A possible mechanism of correlation between plasma vitamin $C$ levels and its beneficial effects may be related to the arterial dilation mediated by the modulation of NO release ${ }^{(252)}$. However, the association between vitamin $\mathrm{C}$ and $\mathrm{HF}$ seems to involve plasma vitamin $\mathrm{C}$ rather than dietary vitamin $\mathrm{C}$, as reported also by the European Investigation into Cancer and Nutrition (EPIC) Norfolk study $^{(253)}$.

In general, supplementation with vitamin $\mathrm{C}$ at dosages between $500 \mathrm{mg}$ and $3 \mathrm{~g} / \mathrm{d}$ is considered safe and well tolerated ${ }^{(254)}$.

Expert opinion. Taking into account the available data in HF settings, no recommendation can be given on the potential usefulness of vitamin $\mathrm{C}$ supplementation. To date no study has also directly investigated the impact of chronic vitamin C supplementation on $\mathrm{HF}$ incidence or prognosis.

\section{Vitamin $E$}

Preclinical models suggest that oxidative stress characterised by the excessive production of reactive oxygen species and reduction of antioxidant defence capacity may play an important role in the pathophysiology of $\mathrm{HF}^{(255)}$. Vitamin $\mathrm{E}$ includes eight distinct chemical entities: $\alpha-, \beta-, \gamma$ - and $\delta$-tocopherol and $\alpha-, \beta$-, $\gamma$ - and $\delta$-tocotrienol. The most studied is $\alpha$-tocopherol, while other forms are poorly understood ${ }^{(256)}$. Vitamin E can protect against oxidative stress by reducing levels of free oxygen radicals and inhibiting LDL oxidation and oxidative cell damage ${ }^{(240)}$. In addition, lipid-lowering activity has been documented through PPAR (PPAR- $\alpha$, PPAR- $\beta$ and PPAR- $\gamma$ ) activation and HMG-COA reductase inhibition ${ }^{(257)}$.

Clinical evidence. In clinical trials, vitamin $\mathrm{E}$ (at doses between 50 and $200 \mathrm{mg}$ ) showed an improvement of endothelial function (reducing the serum levels of hsCRP, advanced glycation endproducts, metalloproteinases and cell adhesion molecules) and arterial stiffness (improving pulse wave velocity, pulse volume and augmentation index $)^{(258,259)}$. The Physician's Health Study II and the Women's Health Study reported no association between vitamin $\mathrm{E}$ supplements and $\mathrm{HF}$ in a primary prevention 
population ${ }^{(260,261)}$ while other studies reported increased risk of HF with vitamin E supplements in those with CVD $^{(262,263)}$, raising concern about the use of vitamin E supplements. In addition, a prospective study of 3919 men aged 60-79 years with no prevalent HF followed up for a mean period of 11 years highlighted that high intake of dietary vitamin $\mathrm{E}$ may be associated with increased HF risk ${ }^{(249)}$. The reason for an increased risk of HF esepcially in older men ${ }^{(264)}$ is still unclear, but it may be related to the fact that $\alpha$-tocopherol may become a pro-oxidant in a preexisting environment with increased oxidative stress, thereby depressing myocardial function. Moreover, $\alpha$-tocopherol may suppress other fat-soluble antioxidants, such as $\gamma$-tocopherol (more powerful antioxidant than $\alpha$-tocopherol), disrupting the natural balance of antioxidant systems and increasing vulnerability to oxidative damage ${ }^{(265)}$. In contrast to dietary vitamin $\mathrm{E}$, no association was observed between plasma vitamin $\mathrm{E}$ and risk of $\mathrm{HF}^{(266)}$ - it is well established that plasma concentrations of vitamin $\mathrm{E}$ increase with the amount of total plasma lipids ${ }^{(267)}$, and particularly with LDL-cholesterol, the principal carrier of tocopherol in the circulation. Thus, vitamin $\mathrm{E}$ absorption may be influenced by factors other than diet and seems to be an unreliable marker of dietary vitamin E ( $\alpha$-tocopherol) intake. This may explain the differences in association between dietary and plasma vitamin $\mathrm{E}$ and $\mathrm{HF}$.

No specific concern has been raised for vitamin E supplementation in the range of the reccomended daily allowance.

Expert opinion. Available epidemiological data and results from RCT do not suggest any benefits from the supplementation of vitamin $\mathrm{E}$ in prevention or as an adjuvant to conventional therapy in $\mathrm{HF}$.

\section{Other minerals and vitamins}

Emerging evidence suggests a pathophysiological role of $\mathrm{Zn}$ and $\mathrm{Cu}$ dyshomeostasis in $\mathrm{HF}$, including promotion of adverse remodelling and clinical deterioration.

Copper. $\mathrm{Cu}$ is a redox-active metal, implicated as an enzymic cofactor in various processes including antioxidant defence mechanisms ( $\mathrm{Cu} / \mathrm{Zn}$-superoxide dismutase, caeruloplasmin), cellular respiration (cytochrome c oxidase), Fe transport (caeruloplasmin), and cross-linking of collagen and elastin (proteinlysine-6-oxidase) ${ }^{(268)}$.

Zinc. Zn, the second most abundant trace element in the body, is involved in gene expression, cell growth and differentiation as a catalytic and structural cofactor. It is implicated in the antioxidant defence and regulation of various metalloproteases (for example, angiotensin-converting enzyme, matrix metalloproteinases, and $\mathrm{Cu} / \mathrm{Zn}$-superoxide dismutase) associated with the healing process, playing a pivotal role in counteracting stress-induced detrimental changes ${ }^{(269)}$. In a study carried out on $125 \mathrm{HF}$ patients and twenty-one healthy volunteers, serum $\mathrm{Cu}$ levels were significantly higher in HFpEF individuals compared with control $(P<0 \cdot 05)$. Additionally, serum $\mathrm{Cu}$ in patients with LVEF $<40 \%$ was significantly higher compared with both controls $(P<0 \cdot 001)$ and HFpEF patients $(P=0 \cdot 003)$. Serum $\mathrm{Zn}$ was significantly lower in acute HF $(P<0.001)$ and HF $(P=0.039)$ compared with control. Serum Cu was increased both in acute chronic HF and correlated with LV systolic and diastolic function. Serum Zn, in contrast, was decreased both in acute HF and chronic HF and independently predicted by clinical status and LV diastolic function ${ }^{(270)}$.

Selenium. Severe Se deficiency is an accepted cause of reversible HF, a condition known as Keshan disease ${ }^{(271)}$. A number of studies have suggested an association between less severe Se deficiency or suboptimal Se status and HF; the majority suggest that patients with HF tend to have lower circulating levels when compared with individuals free from the condition. De Lorgeril et al. $^{\text {(272) }}$ observed a significant positive correlation between blood Se concentration and bicycle exercise stress test maximal oxygen uptake in twenty-one patients with $\mathrm{HF}(r 0 \cdot 9 ; P=0 \cdot 0005)$, suggesting an association between Se status and physical functioning in HF. In addition, in the study by Alehagen et al. ${ }^{(273)}$ regarding healthy elderly Swedish municipality members, cardiovascular mortality was higher in the subgroup with the lower Se concentrations $<65 \mu \mathrm{g} / \mathrm{l}$ in comparison with those having an Se concentration $>85 \mu \mathrm{g} / \mathrm{l}$. A 5 -year prospective randomised double-blind placebo-controlled trial among Swedish citizens aged 70 to 88 years was performed in 443 participants given combined supplementation of Se and CoQ10 or a placebo. During a follow-up time of 5.2 years a significant reduction in cardiovascular mortality was found in the active treatment group $v$. the placebo group (5.9 v. $12.6 \% ; P=0.015)$. NT-proBNP levels were significantly lower in the active group compared with the placebo group (mean values: $214 v .302 \mathrm{ng} / \mathrm{l}$ at 48 months; $P=0.014$ ). In echocardiography a significant better cardiac function score was found in the active supplementation compared with the placebo group $(P=0.03)^{(274)}$.

In summary, patients with HF tend to have lower circulating levels of Se and $\mathrm{Zn}$ and higher levels of $\mathrm{Cu}$, when compared with healthy individuals; however, the prognostic importance and clinical relevance still need to be investigated ${ }^{(275,276)}$.

Riboflavin and pyridoxine. Riboflavin and pyridoxine levels were measured in 100 patients admitted to a hospital with HF, and fifty age- and sex-matched controls. The percentage of individuals with evidence of riboflavin deficiency (erythrocyte glutathione reductase activity coefficient $>1.2$ ) was significantly higher in patients with HF compared with controls $(27.0 \mathrm{v}$. $2.2 \% ; P<0 \cdot 001)$. At the same time, the percentage of patients with pyridoxine deficiency (plasma pyridoxine concentration $<4.9 \mathrm{ng} / \mathrm{ml}(<20 \mathrm{nmol} / \mathrm{l}))$ was also significantly higher in patients with HF $(38.0 v .19 \cdot 0 \% ; P=0 \cdot 02)^{(277)}$. The clinical relevance of these findings has yet to be clarified.

Vitamin $B_{12}$. Observational studies investigating vitamin $\mathrm{B}_{12}$ status in the setting of $\mathrm{HF}$ have demonstrated conflicting results. Some authors suggest no correlation between LVEF and vitamin $\mathrm{B}_{12}$ or folate concentration, in 349 patients undergoing coronary angiography and left ventriculography ${ }^{(278,279)}$. In a larger study carried out on $987 \mathrm{HF}$ patients, vitamin $\mathrm{B}_{12}$ levels were weakly negatively correlated with LVEF $(r-0 \cdot 1$; $P=0 \cdot 015)$ and weakly positively correlated with NT-proBNP 
( $r 0.1 ; P=0.004)$. In addition, mean serum vitamin $\mathrm{B}_{12}$ concentration increased significantly with increasing NYHA class. No relationship was observed between folate status and markers of HF severity ${ }^{(280)}$. An open study without placebo control by Andersson et al. ${ }^{(281)}$ evaluated treatment with pyridoxine $(3 \mathrm{mg} / \mathrm{d})$, folate $(0.8 \mathrm{mg} / \mathrm{d})$ and vitamin $\mathrm{B}_{12}(0.5 \mathrm{mg} / \mathrm{d})$ for 6 weeks in fourteen elderly patients with HF. Treatment was associated with a significant increase in mean plasma folate concentration, but this was not associated with any significant change in levels of NT-proBNP. However, a significant decrease in mean arterial blood pressure and heart rate was observed (95.8 to $90.2 \mathrm{mmHg}$ and 75.0 to 70.0 beats per min, respectively; $P<0.05$ for both) ${ }^{(281)}$. Further studies are still necessary in HF settings.

\section{Discussion and future directions}

In the times of a huge number of supplements and natural products available on the market, it is necessary and critical to present data only for those that have any evidence-based medical data on efficacy and safety in different conditions, such as lipid dis$\operatorname{orders}^{(282,283)}$, hypertension ${ }^{(284)}$, the metabolic syndrome ${ }^{(285)}$ and CVD, including its complication - HF. Only then, remembering that nutraceuticals might be considered only as an addition to optimal therapy, we will avoid overusing these, very often useless preparations; on the other hand, we might help in optimising the applied pharmacotherapy.

Based on the available data it was suggested that a low intake of some nutraceuticals, vitamins and minerals has been associated with an increased risk of developing HF, and supplementation with some nutraceuticals has been reported to improve different parameters related to $\mathrm{HF}$, even on top of guidelinedriven conventional treatment. Nutraceutical components with a potential impact on HF have not yet been deeply investigated. For instance, amino acids (AA) are important constituents of muscle proteins and exert many activities as regulators of both myocardium protein turnover ${ }^{(286,287)}$ and energy metabolism ${ }^{(288)}$. The heart's reliance on AA increases during HF because of high myocardium anabolic activity and cardiomyocyte energy shortage $^{(289)}$. Thus, it has been supposed that the lack of AA intake or absorption could further impair myocardium metabolism in HF patients. In a study involving forty-one patients with clinically stable HF (NYHA class II to IV), compared with controls, HF patients had reduced arterial AA levels, of which both their number and reduced rates were associated with HF severity. Arterial aspartic acid correlated with stroke volume index $(r$ 0.626; $P<0.0001)$ and cardiac index $(r$ 0.424; $P=0.0028)$. The content of arterial aspartic acid $(\mu \mathrm{mol} / \mathrm{l})$ multiplied by the cardiac index was associated with LVEF $(r 0.377$; $P=0 \cdot 0076)$. All NYHA groups had adequate protein intake $(\geq 1 \cdot 1 \mathrm{~g} / \mathrm{kg}$ per $\mathrm{d})$ and inadequate energy intake $(\mathrm{kJ}<$ resting energy expenditure (REE) $\times 1.3$ ) was found only in class IV patients. The study showed that HF patients had reduced arterial AA levels directly related to clinical disease severity and LV dysfunction ${ }^{(290)}$. However, no intervention trial, similarly to other studies with nutraceuticals in HF settings, has yet demonstrated that AA supplementation could have a positive impact on HF.
It has also to be investigated if a combination of nutraceuticals could exert a more relevant impact on HF-related parameters compared with supplementation with a single dietary factor. To date, two studies have measured the effect of a combination multivitamin-multimineral supplement on patients with HF. In the first study, forty-one patients (LVEF $\leq 40 \%$ ) undergoing coronary artery bypass graft were randomised to receive a nutritional supplement containing vitamins, minerals, CoQ10 (150 mg/dose) and carnitine ( $3 \mathrm{~g} /$ dose) or placebo. Treatment with the nutritional supplement was associated with a significant fall in mean LV enddiastolic volume $(170.5 \mathrm{ml} \text { to } 158.9 \mathrm{ml} ; P<0.05)^{(291)}$. Witte et al. ${ }^{(163)}$ observed similar results in a trial involving thirty-three HF subjects randomised to receive a multivitamin-multimineral supplement containing coenzyme Q10 (150 mg/dose) or placebo. After 1 year of treatment, serum levels of folate and vitamin $B_{12}$ increased significantly in the micronutrient group. This was associated with a significant increase in mean LVEF (25.6 to 30.9\%; $P<0.05)$ and a significant increase in mean QoL HF-related tool score (64.4 to $73.9 \% ; P<0.05)$, suggesting a significant QoL improvement ${ }^{(163)}$. Both studies were, however, very small, and so it cannot be concluded that this approach would be a positive suggestion. In fact, this is another main limitation of available data on nutraceutical application in HF patients (relatively small studies, mainly with short-term follow-up), so in most of the cases it is impossible to draw any reliable recommendations.

In conclusion, a growing body of clinical evidence suggests that the intake of adequate dosages of some nutraceuticals (hawthorn extract, CoQ10, L-carnitine, D-ribose, carnosine, vitD, probiotics, $n-3$ PUFA and beet nitrates) might be associated with improvements in self-perceived QoL and/or functional parameters such as EF, stroke volume and cardiac output in HF patients, with minimal or no side effects. Those benefits tended to be greater in earlier HF stages. Efficacy on at least one major cardiovascular event is documented in larger RCT for CoQ10 (Q-SYMBIO trial, 2 years, 420 patients), hawthorn (SPICE trial, 1 year, 2681 patients), $n$-3 PUFA (GISSI-HF, 3.9 years; 6975 patients). For the rest of the above-mentioned nutraceuticals, we still require longer studies and data on their efficacy from the Cardiovascular Outcome Trial. In no case can the use of nutraceuticals be substituted for the consolidated pharmacological treatment of HF.

\section{Acknowledgements}

There is no funding to declare.

A. F. G. C. has given talks, furnished scientific consultancies, and/or participated in trials sponsored by Amgen, Angelini, Boehringer Ingelheim, Meda, Menarini, Merck Sharp \& Dohme, and Sanofi-Synthelabo.

S. v. H. has been a paid consultant to Vifor Pharma, Amgen, AstraZeneca, Bayer, Boehringer Ingelheim, Brahms, Chugai Pharma, Roche and Novartis.

D. V. has given talks and attended conferences sponsored by Bristol-Myers Squibb/Pfizer, Novartis, Servier, Amgen, Bayer and AstraZeneca; and has received speaker fees from Pfizer, Novartis, Servier, Bayer, AstraZeneca and Terapia.

Tomasz Tomasik has received personal fees from Eli Lilly Polska, Boehringer Ingelheim, Novaris and Shire. 
Ž. R. has received honoraria from Sanofi-Aventis.

Peter Penson owns four shares in AstraZeneca PLC. He has received honoraria and/or travel grants.

M. B. has served on the speakers' bureau of Abbott/Mylan, Abbott Vascular, Actavis, Akcea, Amgen, Biofarm, KRKA, Merck Sharp \& Dohme, Sanofi, Servier and Valeant. He has served as a consultant to Abbott Vascular, Akcea, Amgen, Daiichi-Sankyo, Esperion, Lilly, Merck Sharp \& Dohme, Resverlogix and Sanofi, and and has received grants from Sanofi and Valeant.

This review has the official endorsement of: Argentina Lipid Society; Association of Cardiologists of the Republic of Uzbekistan; Association of Preventive Pediatrics of Serbia; Baltic Society of Atherosclerosis; Chinese Atherosclerosis Society; College of Family Physicians in Poland; Croatian Atherosclerosis Society; Czech Society for Atherosclerosis; Egyptian Association for Endocrinology, Diabetes and Atherosclerosis (EAEDA); Egyptian Association of Vascular Biology and Research (EAVA); Estonian Society of Hypertension; Emirates Cardiac Society; Emirates Society of Cardiology; French Atherosclerosis Society; German Atherosclerosis Society; Hellenic Atherosclerosis Society; Hellenic Lipidology Society; Hellenic Society of Lipidology, Atherosclerosis and Vascular Disease; Hungarian Atherosclerosis Society; International Natural Product Sciences Taskforce (INPST); Israeli Society for Treatment and Prevention of Atherosclerosis; Italian Nutraceuticals Society (SINut); Italian Society for Cardiovascular Prevention; Kosovo Society of Cardiology; Kyrgyz Atherosclerosis Society; Latvian Society of Cardiology; Lipid and Atherosclerosis Society of Southern Africa; Lipid and Blood Pressure Meta-analysis Collaboration (LBPMC) Group; Lipid Association of India; Lithuanian Heart Association; Mighty Medic; New French Atherosclerosis Society (NSFA); Polish Lipid Association (PoLA); Romanian National Forum for Prevention; Romanian Society of Cardiology; Russian National Atherosclerosis Society; Saudi Group for Cardiovascular Prevention and Rehabilitation; ScreenPro-FH; Serbian Association for Arteriosclerosis, Thrombosis and Vascular Biology Research (SAATVBR); Slovak Atherosclerosis Association; Slovenian Society of Cardiology; Society of Endocrine and Metabolic Disease of South Africa; Society on Sarcopenia, Cachexia and Wasting Disorders; South African Heart Association; Swiss Society for Familial forms of Hypercholesterolemia (SSFH); Taiwan Society of Lipids and Atherosclerosis; Tunisian Association of Study and Research on Atherosclerosis; Ukrainian Atherosclerosis Society; Venezuelan Society of Atherosclerosis; Very Large Database of Lipids (VLDL).

\section{References}

1. Writing Group Members, Mozaffarian D, Benjamin EJ, et al. (2016) Heart disease and stroke statistics-2016 update: a report from the American Heart Association. Circulation 133, e38-e360.

2. Liu L \& Eisen HJ (2014) Epidemiology of heart failure and scope of the problem. Cardiol Clin 32, 1-8.

3. Bui AL, Horwich TB \& Fonarow GC (2011) Epidemiology and risk profile of heart failure. Nat Rev Cardiol 8, 30-41.

4. Maggioni AP (2015) Epidemiology of heart failure in Europe. Heart Fail Clin 11, 625-635.
5. Meyer S, Brouwers FP, Voors AA, et al. (2015) Sex differences in new-onset heart failure. Clin Res Cardiol 104, 342-350.

6. Kannel WB (2000) Incidence and epidemiology of heart failure. Heart Fail Rev 5, 167-173.

7. Skrzynia C, Berg JS, Willis MS, et al. (2015) Genetics and heart failure: a concise guide for the clinician. Curr Cardiol Rev 11, $10-17$.

8. Yancy CW, Jessup M, Bozkurt B, et al. (2013) 2013 ACCF/AHA guideline for the management of heart failure: a report of the American College of Cardiology Foundation/American Heart Association Task Force on Practice Guidelines. J Am Coll Cardiol 62, e147-e239.

9. McMurray JJ, Adamopoulos S, Anker SD, et al. (2012) ESC Guidelines for the diagnosis and treatment of acute and chronic heart failure 2012: The Task Force for the Diagnosis and Treatment of Acute and Chronic Heart Failure 2012 of the European Society of Cardiology. Developed in collaboration with the Heart Failure Association (HFA) of the ESC. Eur Heart J 33, 1787-1847.

10. Rifai L \& Silver MA (2016) A review of the DASH diet as an optimal dietary plan for symptomatic heart failure. Prog Cardiovasc Dis 58, 548-554.

11. Rifai L, Pisano C, Hayden J, et al. (2015) Impact of the DASH diet on endothelial function, exercise capacity, and quality of life in patients with heart failure. Proc (Bayl Univ Med Cent) 28, 151-156.

12. Tektonidis TG, Åkesson A, Gigante B, et al. (2016) Adherence to a Mediterranean diet is associated with reduced risk of heart failure in men. Eur J Heart Fail 18, 253-259.

13. Salehi-Abargouei A, Maghsoudi Z, Shirani F, et al. (2013) Effects of Dietary Approaches to Stop Hypertension (DASH)-style diet on fatal or nonfatal cardiovascular diseases - incidence: a systematic review and meta-analysis on observational prospective studies. Nutrition 29, 611-618.

14. Wexler R, Pleister A, Raman SV, et al. (2012) Therapeutic lifestyle changes for cardiovascular disease. Phys Sports Med $\mathbf{4 0}$, 109-115.

15. Cicero AF \& Colletti A (2017) Nutraceuticals and dietary supplements to improve quality of life and outcomes in heart failure patients. Curr Pharm Des 23, 1265-1272.

16. Saini R (2011) Coenzyme $\mathrm{Q}_{10}$ : the essential nutrient. J Pharm Bioallied Sci 3, 466-467.

17. Menke T, Niklowitz P, de Sousa G, et al. (2004) Comparison of coenzyme $\mathrm{Q}_{10}$ plasma levels in obese and normal weight children. Clin Chim Acta 349, 121-127.

18. Littarru GP \& Tiano L (2007) Bioenergetic and antioxidant properties of coenzyme $\mathrm{Q}_{10}$ : recent developments. Mol Biotechnol 37, 31-37.

19. Hernández-Camacho JD, Bernier M, López-Lluch G, et al. (2018) Coenzyme $\mathrm{Q}_{10}$ supplementation in aging and disease. Front Physiol 9, 44.

20. Weis M, Mortensen SA, Rassing MR, et al. (1994) Bioavailability of four oral coenzyme $\mathrm{Q}_{10}$ formulations in healthy volunteers. Mol Aspects Med 15, Suppl., 273-280.

21. Miles MV, Horn P, Miles L, et al. (2002) Bioequivalence of coenzyme $\mathrm{Q}_{10}$ from over-the-counter supplements. Nutr Res 22, 919-929.

22. Kumar S, Rao R, Kumar A, et al. (2016) Novel carriers for coenzyme Q10 delivery. Curr Drug Deliv 13, 1184-1204.

23. Bhagavan HN \& Chopra RK (2006) Coenzyme $Q_{10}$ : absorption, tissue uptake, metabolism and pharmacokinetics. Free Radic Res 40, 445-453.

24. Bentinger M, Dallner G, Choknacki T, et al. (2003) Distribution and breakdown of labeled coenzyme $\mathrm{Q}_{10}$ in rat. Free Radic Biol Med 34, 563-575. 
25. López-Lluch G, del Pozo Cruz J, Sánchez-Cuesta A, et al. (2019) Bioavailability of coenzyme Q10 supplements depends on carrier lipids and solubilization. Nutrition 57, 133-140.

26. Weber C, Bysted A \& Hłlmer G (1997) The coenzyme $Q_{10}$ content of the average Danish diet. Int J Vitam Nutr Res 67, 123-129.

27. Onur S, Niklowitz P, Jacobs G, et al. (2015) Association between serum level of ubiquinol and NT-proBNP, a marker for chronic heart failure, in healthy elderly subjects. Biofactors 41, 35-43.

28. Mortensen SA, Rosenfeldt F, Kumar A, et al. (2014) The effect of coenzyme Q10 on morbidity and mortality in chronic heart failure: results from Q-SYMBIO: a randomized double-blind trial. JACC Heart Fail 2, 641-649.

29. Lei L \& Liu Y (2017) Efficacy of coenzyme Q10 in patients with cardiac failure: a meta-analysis of clinical trials. $B M C$ Cardiovasc Disord 17, 196.

30. Fotino AD, Thompson-Paul AM \& Bazzano LA (2013) Effect of coenzyme Q10 supplementation on heart failure: a metaanalysis. Am J Clin Nutr 97, 268-275.

31. Belardinelli R, Mucaj A, Lacalaprice F, et al. (2005) Coenzyme Q10 improves contractility of dysfunctional myocardium in chronic heart failure. Biofactors 25, 137-145.

32. Munkholm H, Hansen HH \& Rasmussen K (1999) Coenzyme Q10 treatment in serious heart failure. Biofactors 9, 285-289.

33. Keogh A, Fenton S, Leslie C, et al. (2003) Randomised double-blind, placebo-controlled trial of coenzyme Q, therapy in class II and III systolic heart failure. Heart Lung Circ 12, 135-141.

34. Langsjoen PH (2000) Lack of effect of coenzyme Q on left ventricular function in patients with congestive heart failure. $J \mathrm{Am}$ Coll Cardiol 35, 816-817.

35. Sander S, Coleman CI, Patel AA, et al. (2006) The impact of coenzyme $\mathrm{Q}_{10}$ on systolic function in patients with chronic heart failure. J Card Fail 12, 464-472.

36. de Frutos F, Gea A, Hernandez-Estefania R, et al. (2015) Prophylactic treatment with coenzyme $Q_{10}$ in patients undergoing cardiac surgery: could an antioxidant reduce complications? A systematic review and meta-analysis. Interact Cardiovasc Thorac Surg 20, 254-259.

37. Mazidi M, Kengne AP \& Banach M (2018) Effects of coenzyme Q10 supplementation on plasma C-reactive protein concentrations: a systematic review and meta-analysis of randomized controlled trials. Pharmacol Res 128, 130-136.

38. Banach M, Serban C, Sahebkar A, et al. (2015) Effects of coenzyme Q10 on statin-induced myopathy: a meta-analysis of randomized controlled trials. Mayo Clin Proc 90, 24-34.

39. Ford E, Adams J \& Graves N (2012) Development of an economic model to assess the cost-effectiveness of hawthorn extract as an adjunct treatment for heart failure in Australia. BMJ Open 2, e001094.

40. Koch E \& Malek FA (2011) Standardized extracts from hawthorn leaves and flowers in the treatment of cardiovascular disorders - preclinical and clinical studies. Planta Med 77, $1123-1128$

41. Wang J, Xiong X \& Feng B (2013) Effect of Crataegus usage in cardiovascular disease prevention: an evidence-based approach. Evid Based Complement Alternat Med 2013, 149363.

42. Schwinger RH, Pietsch M, Frank K, et al. (2000) Crataegus special extract WS 1442 increases force of contraction in human myocardium cAMP-independently. J Cardiovasc Pharmacol 35, 700-707.

43. Münch G, Brixius K, Frank K, et al. (1997) WS 1442 (extract of Crataegus species) increases force of contraction in human failing myocardium by inhibition of the $\mathrm{Na}^{+} / \mathrm{K}^{+}$-ATPase. Circulation 96, Suppl., I-729.

44. Krzeminski T \& Chatterjee SS (1993) Ischemia and early reperfusion induced arrythmias: beneficial effects of an extract of Crataegus oxyacantha L. Pharm Pharmacol Lett 3, 45-48.

45. Willer EA, Malli R, Bondarenko AI, et al. (2012) The vascular barrier protecting hawthorn extract WS® 1442 raises endothelial calcium levels by inhibition of SERCA and activation of the IP3 pathway. J Mol Cell Cardiol 53, 567-577.

46. Holubarsch CJ, Colucci WS, Meinertz T, et al. (2000) Survival and Prognosis: Investigation of Crataegus Extract WS 1442 in congestive heart failure (SPICE) - rationale, study design and study protocol. Eur J Heart Fail 2, 431-437.

47. Holubarsch CJ, Colucci WS, Meinertz T, et al. (2008) The efficacy and safety of Crataegus extract WS 1442 in patients with heart failure: the SPICE trial. Eur J Heart Fail 10, 1255-1263.

48. Tauchert M (2002) Efficacy and safety of Crataegus extract WS 1442 in comparison with placebo in patients with chronic stable New York Heart Association class-III heart failure. Am Heart J 143, 910-915.

49. Pittler MH, Guo R \& Ernst E (2008) Hawthorn extract for treating chronic heart failure. Cochrane Database Syst Rev, issue 1, CD005312.

50. Eggeling T, Regitz-Zagrosek V, Zimmermann A, et al. (2011) Baseline severity but not gender modulates quantified Crataegus extract effects in early heart failure - a pooled analysis of clinical trials. Phytomedicine 18, 1214-1219.

51. Daniele C, Mazzanti G, Pittler MH, et al. (2006) Adverse-event profile of Crataegus spp.: a systematic review. Drug Saf $\mathbf{2 9}$, 523-535.

52. Khairallah RJ, Sparagna GC, Khanna N, et al. (2010) Dietary supplementation with docosahexaenoic acid, but not eicosapentaenoic acid, dramatically alters cardiac mitochondrial phospholipid fatty acid composition and prevents permeability transition. Biochim Biophys Acta 1797, 1555-1562.

53. Khairallah RJ, Kim J, O'Shea KM, et al. (2012) Improved mitochondrial function with diet induced increase in either docosahexaenoic acid or arachidonic acid in membrane phospholipids. PLOS ONE 7, e34402.

54. O'Shea KM, Khairallah RJ, Sparagna GC, et al. (2009) Dietary $\omega$-3 fatty acids alter cardiac mitochondrial phospholipid composition and delay $\mathrm{Ca}^{2+}$ induced permeability transition. J Mol Cell Cardiol 47, 819-827.

55. Galvao TF, Khairallah RJ, Dabkowski ER, et al. (2013) Marine n3 polyunsaturated fatty acids enhance resistance to mitochondrial permeability transition in heart failure, but do not improve survival. Am J Physiol Heart Circ Physiol 73, H12-H21.

56. Stanley WC, Khairallah RJ \& Dabkowski ER (2012) Update on lipids and mitochondrial function: impact of dietary $n-3$ polyunsaturated fatty acids. Curr Opin Clin Nutr Metab Care 15, 122-126.

57. Chrysohoou C, Metallinos G, Georgiopoulos G, et al. (2016) Short term omega-3 polyunsaturated fatty acid supplementation induces favorable changes in right ventricle function and diastolic filling pressure in patients with chronic heart failure: a randomized clinical trial. Vascul Pharmacol 79, 43-50.

58. Djoussé L, Akinkuolie AO, Wu JH, et al. (2012) Fish consumption, omega-3 fatty acids and risk of heart failure: a metaanalysis. Clin Nutr 31, 846-853.

59. Tavazzi L, Maggioni AP, Marchioli R, et al. (2008) Effect of $n-3$ polyunsaturated fatty acids in patients with chronic heart failure (the GISSI-HF trial): a randomised, double-blind, placebocontrolled trial. Lancet 372, 1223-1230.

60. Ghio S, Scelsi L, Latini R, et al. (2010) Effects of $n$-3 polyunsaturated fatty acids and of rosuvastatin on left ventricular function 
in chronic heart failure: a substudy of GISSI-HF trial. Eur J Heart Fail 12, 1345-1353.

61. Maki KC, Palacios OM, Bell M, et al. (2017) Use of supplemental long-chain omega-3 fatty acids and risk for cardiac death: an updated meta-analysis and review of research gaps. J Clin Lipidol 11, 1152-1160.e2.

62. Moertl D, Hammer A, Steiner S, et al. (2011) Dose-dependent effects of omega-3-polyunsaturated fatty acids on systolic left ventricular function, endothelial function, and markers of inflammation in chronic heart failure of non ischemic origin: a double-blind, placebo-controlled, 3-arm study. Am Heart J 161, 915.e1-915.e9.

63. Dabkowski ER, O'Connell KA, Xu W, et al. (2013) Docosahexaenoic acid supplementation alters key properties of cardiac mitochondria and modestly attenuates development of left ventricular dysfunction in pressure overloadinduced heart failure. Cardiovasc Drugs Ther 27, 499-510.

64. Oikonomou E, Vogiatzi G, Karlis D, et al. (2019) Effects of omega-3 polyunsaturated fatty acids on fibrosis, endothelial function and myocardial performance, in ischemic heart failure patients. Clin Nutr 38, 1188-1197.

65. Mehra MR, Lavie CJ, Ventura HO, et al. (2006) Fish oils produce anti-inflammatory effects and improve body weight in severe heart failure. J Heart Lung Transplant 25, 834-838.

66. Pepe S \& McLennan PL (2002) Cardiac membrane fatty acid composition modulates myocardial oxygen consumption and postischemic recovery of contractile function. Circulation 105, 2303-2308.

67. Ponikowski P, Voors AA, Anker SD, et al. (2016) 2016 ESC Guidelines for the diagnosis and treatment of acute and chronic heart failure: The Task Force for the diagnosis and treatment of acute and chronic heart failure of the European Society of Cardiology (ESC). Developed with the special contribution of the Heart Failure Association (HFA) of the ESC. Eur J Heart Fail 18, 891-975.

68. Cicero AF, Reggi A, Parini A, et al. (2012) Application of polyunsaturated fatty acids in internal medicine: beyond the established cardiovascular effects. Arch Med Sci 8, 784-793.

69. Omar S, Vande Hei AG, Battisha A, et al. (2019) Cardiovascular, electrophysiologic, and hematologic effects of omega-3 fatty acids beyond reducing hypertriglyceridemia: as it pertains to the recently published REDUCE-IT trial. Cardiovasc Diabetol 18, 84.

70. El-Hattab AW \& Scaglia F (2015) Disorders of carnitine biosynthesis and transport. Mol Genet Metab 116, 107-112.

71. Katz AM (1998) Is the failing heart energy depleted? Cardiol Clinics 16, 633-644.

72. Flanagan JL, Simmons PA, Vehige J, et al. (2010) Role of carnitine in disease. Nutr Metab (Lond) 7, 30.

73. Blanca AJ, Ruiz-Armenta MV, Zambrano S, et al. (2016) Inflammatory and fibrotic processes are involved in the cardiotoxic effect of sunitinib: protective role of L-carnitine. Toxicol Lett 241, 9-18.

74. Omori Y, Ohtani T, Sakata Y, et al. (2012) L-Carnitine prevents the development of ventricular fibrosis and heart failure with preserved ejection fraction in hypertensive heart disease. J Hypertens 30, 1834-1844.

75. Song X, Qu H, Yang Z, et al. (2017) Efficacy and safety of L-carnitine treatment for chronic heart failure: a meta-analysis of randomized controlled trials. Biomed Res Int 2017, 6274854 .

76. Mazidi M, Rezaie P \& Banach M (2017) Impact of L-carnitine on C-reactive protein: a systematic review and meta-analysis of 10 randomized control trials with 925 patients. Presentation at the 2nd CPPEI Congress in Vienna, July 2017.
77. Pooyandjoo M, Nouhi M, Shab-Bidar S, et al. (2016) The effect of (L-) carnitine on weight loss in adults: a systematic review and meta-analysis of randomized controlled trials. Obes Rev 17, 970-976.

78. Serban MC, Sahebkar A, Mikhailidis DP, et al. (2016) Impact of L-carnitine on plasma lipoprotein(a) concentrations: a systematic review and meta-analysis of randomized controlled trials. Sci Rep 6, 19188.

79. Kattoor AJ, Goel A \& Mehta JL (2018) Thiamine therapy for heart failure: a promise or fiction? Cardiovasc Drugs Ther 32, 313-317.

80. Lonsdale D (2018) Thiamin. Adv Food Nutr Res 83, 1-56.

81. Anderson S, Vickery C \& Nicol A (1986) Adult thiamine requirements and the continuing need to fortify processed cereals. Lancet 328, 85-89.

82. Kerns JC, Cherinne Arundel C \& Chawla LS (2015) Thiamin deficiency in people with obesity. Adv Nutr 6, 147-153.

83. Oliveira FA, Guatimosim S, Castro CH, et al. (2007) Abolition of reperfusion-induced arrhythmias in hearts from thiaminedeficient rats. Am J Phys Heart Circ Phys 293, H394-H401.

84. Jain A, Mehta R, Al-Ani M, et al. (2015) Determining the role of thiamine deficiency in systolic heart failure: a meta-analysis and systematic review. J Card Fail 21, 1000-1007.

85. Katta N, Balla S \& Alpert MA (2016) Does long-term furosemide therapy cause thiamine deficiency in patients with heart failure? A focused review. Am J Med 129, 753.e7-753.e11.

86. Jikrona R, Suharjono S \& Ahmad A (2017) Thiamine supplement therapy improves ejection fraction value in stage II heart failure patients. Folia Medica Indon 53, 139-143.

87. Shimon H, Almog S, Vered Z, et al. (1995) Improved left ventricular function after thiamine supplementation in patients with congestive heart failure receiving long-term furosemide therapy. Am J Med 98, 485-490.

88. Schoenenberger A, Schoenenberger-Berzins R, der Maur C, et al. (2012) Thiamine supplementation in symptomatic chronic heart failure: a randomized, double-blind, placebocontrolled, cross-over pilot study. Clin Res Cardiol 101, 159-164.

89. Mousavi M, Namazi S, Avadi M, et al. (2017) Thiamine supplementation in patients with chronic heart failure receiving optimum medical treatment. J Cardiol Curr Res 9, 00316.

90. Rocha RM, Silva GV, de Albuquerque DC, et al. (2008) Influence of spironolactone therapy on thiamine blood levels in patients with heart failure. Arq Bras Cardiol 90, 324-328.

91. Fernández-Murga L, Tarín JJ, García-Perez MA, et al. (2011) The impact of chocolate on cardiovascular health. Maturitas 69, 312-321.

92. Rusconi M \& Conti A (2010) Theobroma cacao L., the food of the gods: a scientific approach beyond myths and claims. Pharmacol Res 61, 5-13.

93. Ortega N, Romero MP, Macià A, et al. (2008) Obtention and characterization of phenolic extracts from different cocoa sources. J Agric Food Chem 56, 9621-9627.

94. Schroeter H, Heiss C, Balzer J, et al. (2006) (-)-Epicatechin mediates beneficial effects of flavanol-rich cocoa on vascular function in humans. Proc Natl Acad Sci US A 103, 1024-1029.

95. Heiss C, Jahn S, Taylor M, et al. (2010) Improvement of endothelial function with dietary flavanols is associated with mobilization of circulating angiogenic cells in patients with coronary artery disease. J Am Coll Cardiol 56, 218-224.

96. Steinhaus DA, Mostofsky E, Levitan EB, et al. (2017) Chocolate intake and incidence of heart failure: findings from the cohort of Swedish men. Am Heart J 183, 18-23.

97. Petrone AB, Gaziano JM \& Djousse L (2014) Chocolate consumption and risk of heart failure in the Physicians' Health Study. Eur J Heart Fail 16, 1372-1376. 
98. Alagiakrishnan K, Banach M, Ahmed A, et al. (2016) Complex relationship of obesity and obesity paradox in heart failure higher risk of developing heart failure and better outcomes in established heart failure. Ann Med 48, 603-613.

99. De Palma R, Sotto I, Wood EG, et al. (2016) Cocoa flavanols reduce N-terminal pro-B-type natriuretic peptide in patients with chronic heart failure. ESC Heart Fail 3, 97-106.

100. Flammer AJ, Sudano I, Wolfrum M, et al. (2012) Cardiovasculareffects of flavanol-rich chocolate in patients with heart failure. Eur Heart J 33, 2172-2180.

101. Taub PR, Ramirez-Sanchez I, Patel M, et al. (2016) Beneficial effects of dark chocolate on exercise capacity in sedentary subjects: underlying mechanisms. A double blind, randomized, placebo controlled trial. Food Funct 7, 3686-3693.

102. Boldyrev AA, Aldini G \& Derave W (2013) Physiology and pathophysiology of carnosine. Physiol Rev 93, 1803-1845.

103. Sale C, Saunders B \& Harris RC (2010) Effect of beta-alanine supplementation on muscle carnosine concentrations and exercise performance. Amino Acids 39, 321-333.

104. Artioli GG, Gualano B, Smith A, et al. (2010) Role of betaalanine supplementation on muscle carnosine and exercise performance. Med Sci Sports Exerc 42, 1162-1173.

105. Kohen R, Yamamoto Y, Cundy KC, et al. (1988) Antioxidant activity of carnosine, homocarnosine, and anserine present in muscle and brain. Proc Natl Acad Sci U S A 85, 3175-3179.

106. Pavlov AR, Revina AA, Dupin AM, et al. (1993) The mechanism of interaction of carnosine with superoxide radicals in water solutions. Biochim Biophys Acta 1157, 304-312.

107. Kang JH, Kim KS, Choi SY, et al. (2002) Protective effects of carnosine, homocarnosine and anserine against peroxyl radical-mediated $\mathrm{Cu}, \mathrm{Zn}$-superoxide dismutase modification. Biochim Biophys Acta 1570, 89-96.

108. Seddon M, Looi YH \& Shah AM (2007) Oxidative stress and redox signalling in cardiac hypertrophy and heart failure. Heart 93, 903-907.

109. Zaloga GP, Roberts PR, Black KW, et al. (1997) Carnosine is a novel peptide modulator of intracellular calcium and contractility in cardiac cells. Am J Physiol 272, H462-H468.

110. Bokeriya LA, Boldyrev AA, Movsesyan RR, et al. (2008) Cardioprotective effect of histidine-containing dipeptides in pharmacological cold cardioplegia. Bull Exp Biol Med 145, 323-327.

111. Lombardi C, Carubelli V, Lazzarini V, et al. (2014) Effects of oral amino acid supplements on functional capacity in patients with chronic heart failure. Clin Med Insights Cardiol 8, 39-44.

112. Rosenfeldt FL (1998) Metabolic supplementation with orotic acid and magnesium orotate. Cardiovasc Drugs Ther 12, Suppl. 2, 147-152.

113. Stepura OB \& Martynow AI (2009) Magnesium orotate in severe congestive heart failure (MACH). Int J Cardiol 134, $145-147$.

114. McCarty MF \& Di Nicolantonio JJ (2014) $\beta$-Alanine and orotate as supplements for cardiac protection. Open Heart 1, e000119.

115. Décombaz J, Beaumont M, Vuichoud J, et al. (2012) Effect of slow-release $\beta$-alanine tablets on absorption kinetics and paresthesia. Amino Acids 43, 67-76.

116. Holick MF (2007) Vitamin D deficiency. N Engl J Med 357, 266-281.

117. Wang Y, Zhu J \& DeLuca HF (2012) Where is the vitamin D receptor? Arch Biochem Biophys 523, 123-133.

118. O'Connell TD \& Simpson RU (1996) Immunochemical identification of the 1,25-dihydroxyvitamin $\mathrm{D}_{3}$ receptor protein in human heart. Cell Biol Int 20, 621-624.
119. Simpson RU, Thomas GA \& Arnold AJ (1985) Identification of 1,25-dihydroxyvitamin $\mathrm{D}_{3}$ receptors and activities in muscle. $J$ Biol Chem 260, 8882-8891.

120. Pourdjabbar A, Dwivedi G \& Haddad H (2013) The role of vitamin D in chronic heart failure. Curr Opin Cardiol 28, 216-222.

121. Sun Y, Zhang J, Zhang JQ, et al. (2000) Local angiotensin II and transforming growth factor- $\beta 1$ in renal fibrosis of rats. Hypertension 35, 1078-1084.

122. Mann DL (2002) Inflammatory mediators and the failing heart: past, present, and the foreseeable future. Circ Res $\mathbf{9 1}$, 988-998.

123. Li YC (2012) Vitamin D: roles in renal and cardiovascular protection. Curr Opin Nephrol Hypertens 21, 72-79.

124. Levin A, Bakris GL, Molitch M, et al. (2007) Prevalence of abnormal serum vitamin D, PTH, calcium, and phosphorus in patients with chronic kidney disease: results of the study to evaluate early kidney disease. Kidney Int 71, 31-38.

125. de Zeeuw D, Agarwal R, Amdahl M, et al. (2010) Selective vitamin D receptor activation with paricalcitol for reduction of albuminuria in patients with type 2 diabetes (VITAL study): a randomised controlled trial. Lancet 376, 1543-1551.

126. Keane WF \& Eknoyan G (1999) Proteinuria, albuminuria, risk, assessment, detection, elimination (PARADE): a position paper of the National Kidney Foundation. Am J Kidney Dis 33, 1004-1010.

127. Brenner BM, Cooper ME, de Zeeuw D, et al. (2001) Effects of losartan on renal and cardiovascular outcomes in patients with type 2 diabetes and nephropathy. NEngl J Med 345, 861-869.

128. Liu LC, Voors AA, van Veldhuisen DJ, et al. (2011) Vitamin D status and outcomes in heart failure patients. Eur J Heart Fail 13, 619-625.

129. Gullestad L, Ueland T, Vinge LE, et al. (2012) Inflammatory cytokines in heart failure: mediators and markers. Cardiology 122, 23-35.

130. Hedayat M, Mahmoudi MJ, Rose NR, et al. (2010) Proinflammatory cytokines in heart failure: double-edged swords. Heart Fail Rev 15, 543-562.

131. Haudek SB, Taffet GE, Schneider MD, et al. (2007) TNF provokes cardiomyocyte apoptosis and cardiac remodeling through activation of multiple cell death pathways. $J$ Clin Invest 117, 2692-2701.

132. Upadhyay A, Larson MG, Guo CY, et al. (2011) Inflammation, kidney function and albuminuria in the Framingham Offspring cohort. Nephrol Dial Transplant 26, 920-926.

133. Yu X, Yang Z \& Yu M (2010) Correlation of tumor necrosis factor alpha and interleukin 6 with hypertensive renal damage. Ren Fail 32, 475-479.

134. Mora JR, Iwata M \& von Andrian UH (2008) Vitamin effects on the immune system: vitamins A and D take centre stage. Nat Rev Immunol 8, 685-698.

135. Li YY, Feng YQ, Kadokami T, et al. (2000) Myocardial extracellular matrix remodeling in transgenic mice overexpressing tumor necrosis factor $\alpha$ can be modulated by antitumor necrosis factor $\alpha$ therapy. Proc Natl Acad Sci U S A 97 , $12746-12751$.

136. Gunja-Smith Z, Morales AR, Romanelli R, et al. (1996) Remodeling of human myocardial collagen in idiopathic dilated cardiomyopathy. Role of metalloproteinases and pyridinoline cross-links. Am J Pathol 148, 1639-1648.

137. Bielecka-Dabrowa A, Sakowicz A, Pietrucha T, et al. (2017) The profile of selected single nucleotide polymorphisms in patients with hypertension and heart failure with preserved and mid-range ejection fraction. Sci Rep 7, 8974. 
138. Faridi KF, Lupton JR, Martin SS, et al. (2017) Vitamin D deficiency and non-lipid biomarkers of cardiovascular risk. Arch Med Sci 13, 732-737.

139. Kim DH, Sabour S, Sagar UN, et al. (2008) Prevalence of hypovitaminosis $\mathrm{D}$ in cardiovascular diseases (from the National Health and Nutrition Examination Survey 2001 to 2004). Am J Cardiol 102, 1540-1544.

140. Anderson JL, May HT, Horne BD, et al. (2010) Relation of vitamin D deficiency to cardiovascular risk factors, disease status, and incident events in a general health care population. $\mathrm{AmJ}$ Cardiol 106, 963-968.

141. Pilz S, Marz W, Wellnitz B, et al. (2008) Association of vitamin $\mathrm{D}$ deficiency with heart failure and sudden cardiac death in a large cross-sectional study of patients referred for coronary angiography. J Clin Endocrinol Metab 93, 3927-3935.

142. Zittermann A, Schleithoff SS, Tenderich G, et al. (2003) Low vitamin D status: a contributing factor in the pathogenesis of congestive heart failure? J Am Coll Cardiol 41, 105-112.

143. Ameri P, Ronco D, Casu M, et al. (2010) High prevalence of vitamin D deficiency and its association with left ventricular dilation: an echocardiography study in elderly patients with chronic heart failure. Nutr Metab Cardiovasc Dis 20, 633-640.

144. Iqba N, Ducharme J, Desai S, et al. (2008) Status of bone mineral density in patients selected for cardiac transplantation. Endocr Pract 14, 704-712.

145. Alsafwah S, Laguardia SP \& Nelson MD (2008) Hypovitaminosis $\mathrm{D}$ in African Americans residing in Memphis, Tennessee with and without heart failure. Am J Med Sci 335, 292-297.

146. Arroyo M, Laguardia SP, Bhattacharya SK, et al. (2006) Micronutrients in African-Americans with decompensated and compensated heart failure. Transl Res 148, 301-308.

147. Shane E, Mancini D, Aaronson K, et al. (1997) Bone mass, vitamin D deficiency, and hyperparathyroidism in congestive heart failure. Am J Med 103, 197-207.

148. Boxer RS, Dauser DA, Walsh SJ, et al. (2008) The association between vitamin $\mathrm{D}$ and inflammation with the 6-minute walk and frailty in patients with heart failure. J Am Geriatr Soc 56, 454-461.

149. Boxer RS, Kenny AM, Cheruvu VK, et al. (2010) Serum 25-hydroxyvitamin D concentration is associated with functional capacity in older adults with heart failure. $\mathrm{Am}$ Heart J 160, 893-899.

150. Zittermann A, Schleithoff SS, Gotting C, et al. (2008) Poor outcome in end stage heart failure patients with low circulating calcitriol levels. Eur J Heart Fail 10, 321-327.

151. Schleithoff SS, Zittermann A, Tenderich G, et al. (2006) Vitamin D supplementation improves cytokine profiles in patients with congestive heart failure: a double-blind, randomized, placebo-controlled trial. Am J Clin Nutr 83, 754-759.

152. Shedeed SA (2012) Vitamin D supplementation in infants with chronic congestive heart failure. Pediatr Cardiol 33, 713-719.

153. Gotsman I, Shauer A, Zwas DR, et al. (2012) Vitamin D deficiency is a predictor of reduced survival in patients with heart failure; vitamin D supplementation improves outcome. Eur J Heart Fail 14, 357-366.

154. Witham MD, Crighton LJ, Gillespie ND, et al. (2010) The effects of vitamin D supplementation on physical function and quality of life in older patients with heart failure: a randomized controlled trial. Circ Heart Fail 3, 195-201.

155. Razzaque MS (2018) Can adverse effects of excessive vitamin D supplementation occur without developing hypervitaminosis D? J Steroid Biochem Mol Biol 180, 81-86.

156. Robien K, Oppeneer SJ, Kelly JA, et al. (2013) Drug-vitamin D interactions: a systematic review of the literature. Nutr Clin Pract 28, 194-208.
157. Rude RK (1989) Physiology of magnesium metabolism and the important role of magnesium in potassium deficiency. $A m \mathrm{~J}$ Cardiol 63, 31-34.

158. Gattlieb SS (1989) Importance of magnesium in congestive heart failure. Am J Cardiol 63, 39-42.

159. Ralston MA, Mumane MR, Unverferth DV, et al. (1990) Serum and tissue magnesium concentrations in patients with heart failure and serious ventricular arrhythmias. Ann Intern Med 113, 841-846.

160. Douban S, Brodsky MA, Whang DD, et al. (1996) Significance of magnesium congestive heart failure. Am Heart $J$ 132, 664-671.

161. Wester PO (1992) Electrolyte balance in heart failure and the role of magnesium ions. Am J Cardiol 70, 44-49.

162. Wu X, Ackermann U \& Sonnenberg H (1995) Potassium depletion and salt-sensitive hypertension in DAHL rats: effect on calcium, magnesium, and phosphate excretions. Clin Exp Hypertens 17, 989-1008.

163. Witte KKA, Nikitin NP, Parker AC, et al. (2005) The effect of micronutrient supplementation on quality-of-life and left ventricular function in elderly patients with chronic heart failure. Eur Heart J 26, 2238-2244.

164. Almoznino-Sarafian D, Sarafian G, Berman S, et al. (2009) Magnesium administration may improve heart rate variability in patients with heart failure. Nutr Metab Cardiovasc Dis 19, 641-645.

165. He K, Liu K, Daviglus ML, et al. (2006) Magnesium intake and incidence of metabolic syndrome among young adults. Circulation 113, 1675-1682.

166. Song Y, He K, Levitan EB, et al. (2006) Effects of oral magnesium supplementation on glycaemic control in type 2 diabetes: a meta-analysis of randomized double-blind controlled trials. Diabetes Med 23, 1050-1056.

167. Lee SH, Miller ER, Guallar E, et al. (2002) The effect of magnesium supplementation on blood pressure: a metaanalysis of randomized clinical trials. Am J Hypertens $\mathbf{1 5}$, 691-696.

168. Misialek JR, Lopez FL, Lutsey PL, et al. (2013) Serum and dietary magnesium and incidence of atrial fibrillation in whites and in African Americans; Atherosclerosis Risk in Communities (ARIC) Study. Circ J 77, 323-329.

169. Lutsey PL, Alonso A, Michos ED, et al. (2014) Serum magnesium, phosphorus, and calcium are associated with risk of incident heart failure: the Atherosclerosis Risk in Communities (ARIC) Study. Am J Clin Nutr 100, 756-764.

170. Altura BM \& Altura BT (1986) Biochemistry and pathophysiology of congestive heart failure: is there a role for magnesium? Magnesium 5, 134-143.

171. Adamopoulos C, Pitt B, Sui X, et al. (2009) Low serum magnesium and cardiovascular mortality in chronic heart failure: a propensity-matched study. Int J Cardiol 136, 270-277.

172. Wannamethee SG, Papacosta O, Lennon L, et al. (2018) Serum magnesium and risk of incident heart failure in older men: The British Regional Heart Study. Eur J Epidemiol 33, 873-882.

173. Angkananard T, Anothaisintawee T, Eursiriwan S, et al. (2016) The association of serum magnesium and mortality outcomes in heart failure patients: a systematic review and metaanalysis. Medicine (Baltimore) 95, e5406.

174. Taveira TH, Ouellette D, Gulum A, et al. (2016) Relation of magnesium intake with cardiac function and heart failure hospitalizations in black adults: The Jackson Heart Study. Circ Heart Fail 9, e002698.

175. Song EK \& Kang SM (2017) Micronutrient deficiency independently predicts adverse health outcomes in patients with heart failure. J Cardiovasc Nurs 32, 47-53. 
176. Mazidi M, Rezaie P \& Banach M (2018) Effect of magnesium supplements on serum C-reactive protein: a systematic review and meta-analysis. Arch Med Sci 14, 707-716.

177. Lopez FL, Agarwal SK, Grams ME, et al. (2013) Relation of serum phosphorus levels to the incidence of atrial fibrillation (from the Atherosclerosis Risk in Communities [ARIC] Study). Am J Cardiol 111, 857-862.

178. Khan AM, Lubitz SA, Sullivan LM, et al. (2013) Low serum magnesium and the development of atrial fibrillation in the community: the Framingham Heart Study. Circulation 127, 33-38.

179. Wang TJ, Larson MG, Levy D, et al. (2003) Temporal relations of atrial fibrillation and congestive heart failure and their joint influence on mortality: the Framingham Heart Study. Circulation 107, 2920-2925.

180. Chamberlain AM, Redfield MM, Alonso A, et al. (2011) Atrial fibrillation and mortality in heart failure: a community study. Circ Heart Fail 4, 740-746.

181. Miller S, Crystal E, Garfinkle M, et al. (2005) Effects of magnesium on atrial fibrillation after cardiac surgery: a meta-analysis. Heart 91, 618-623.

182. Ho KM \& Lewis JP (2010) Prevention of atrial fibrillation in cardiac surgery: time to consider a multimodality pharmacological approach. Cardiovasc Ther 28, 59-65.

183. Banach M, Kourliouros A, Reinhart KM, et al. (2010) Postoperative atrial fibrillation - what do we really know? Curr Vasc Pharmacol 8, 553-572.

184. Rude RK (2010) Magnesium. In Encyclopedia of Dietary Supplements, pp. 527-537 [PM Coates, JM Betz, MR Blackman and GM Cragg, editors]. New York: Informa Healthcare.

185. Fang X, Wang K, Han D, et al. (2016) Dietary magnesium intake and the risk of cardiovascular disease, type 2 diabetes, and all-cause mortality: a dose-response meta-analysis of prospective cohort studies. BMC Med 14, 210.

186. Urso C, Brucculeri S \& Caimi G (2015) Acid-base and electrolyte abnormalities in heart failure: pathophysiology and implications. Heart Fail Rev 20, 493-503.

187. Lima B, Forrester MT, Hess DT, et al. (2010) S-nitrosylation in cardiovascular signaling. Circ Res 106, 633-646.

188. Coggan AR \& Peterson LR (2016) Dietary nitrate and skeletal muscle contractile function in heart failure. Curr Heart Fail Rep 13, 158-165.

189. Hare JM \& Stamler JS (2005) NO/redox disequilibrium in the failing heart and cardiovascular system. J Clin Invest 115, 509-517.

190. Katz SD (1995) The role of endothelium-derived vasoactive substances in the pathophysiology of exercise in tolerance in patients with congestive heart failure. Prog Cardiovasc Dis 38, 23-50.

191. Kapil V, Weitzberg E \& Lundberg JO (2014) Clinical evidence demonstrating the utility of inorganic nitrate in cardiovascular health. Nitric Oxide 38, 45-57.

192. Cosby K, Partovi KS, Crawford JH, et al. (2003) Nitrite reduction to nitric oxide by deoxyhemoglobin vasodilates the human circulation. Nature Med 9, 1498-1505.

193. Lundberg JO, Carlstrom M, Larsen FJ, et al. (2011) Roles of dietary inorganic nitrate in cardiovascular health and disease. Cardiovasc Res 89, 525-532.

194. Omar SA, Artime E \& Webb AJ (2012) A comparison of organic and inorganic nitrates/nitrites. Nitric Oxide 26, 229-240.

195. Redfield MM, Anstrom KJ, Levine JA, et al. (2015) Isosorbide mononitrate in heart failure with preserved ejection fraction. N Engl J Med 373, 2314-2324.

196. Miller GD, Marsh AP, Dove RW, et al. (2012) Plasma nitrate and nitrite are increased by a high-nitrate supplement but not by high-nitrate foods in older adults. Nutr Res 32, 160-168.
197. Lansley K, Winyard P, Fulford J, et al. (2011) Dietary nitrate supplementation reduces the $\mathrm{O}_{2}$ cost of walking and running: a placebo-controlled study. J Appl Physiol 110, 591-600.

198. Bailey SJ, Winyard P, Vanhatalo A, et al. (2009) Dietary nitrate supplementation reduces the $\mathrm{O}_{2}$ cost of low-intensity exercise and enhances tolerance to high-intensity exercise in humans. J Appl Physiol 107, 1144-1155.

199. Hoon MW, Johnson NA, Chapman PG, et al. (2014) The effect of nitrate supplementation on exercise performance in healthy individuals: a systematic review and meta-analysis. Int J Sport Nutr Exerc Metab 23, 522-532.

200. Zamani P, Rawat D, Shiva-Kumar P, et al. (2015) Effect of inorganic nitrate on exercise capacity in heart failure with preserved ejection fraction. Circulation 131, 371-380.

201. Kelly J, Fulford J, Vanhatalo A, et al. (2013) Effects of short-term dietary nitrate supplementation on blood pressure, $\mathrm{O}_{2}$ uptake kinetics, and muscle and cognitive function in older adults. Am J Physiol Regul Integr Comp Physiol 304, R73-R83.

202. Coggan AR, Leibowitz JL, Spearie CA, et al. (2015) Acute dietary nitrate intake improves muscle contractile function in patients with heart failure: a double-blind, placebocontrolled, randomized trial. Circ Heart Fail 85, 914-920.

203. Eggebeen J, Kim-Shapiro DB, Haykowsky M, et al. (2016) One week of daily dosing with beetroot juice improves submaximal endurance and blood pressure in older patients with heart failure and preserved ejection fraction. JACC Heart Fail $\mathbf{4}$, 428-437.

204. Coggan AR, Broadstreet SR \& Mahmood K (2018) Dietary nitrate increases $\mathrm{VO}_{2}$ peak and performance but does not alter ventilation or efficiency in patients with heart failure with reduced ejection fraction. J Card Fail 24, 65-73.

205. Shaltout HA, Eggebeen J, Marsh AP, et al. (2017) Effects of supervised exercise and dietary nitrate in older adults with controlled hypertension and/or heart failure with preserved ejection fraction. Nitric Oxide 69, 78-90.

206. Kenjale AA, Ham KL, Stabler T, et al. (2011) Dietary nitrate supplementation enhances exercise performance in peripheral arterial disease. J Appl Physiol 110, 1582-1591.

207. Bahadoran Z, Mirmiran P, Kabir A, et al. (2017) The nitrateindependent blood pressure-lowering effect of beetroot juice: a systematic review and meta-analysis. Adv Nutr 8, 830-838.

208. Pauly DF \& Pepine CJ (2000) D-Ribose as a supplement for cardiac energy metabolism. J Cardiovasc Pharmacol Therapeut 5, 249-258.

209. Kriett JM, Ward HB \& Bianco RW (1983) Recovery of adenine nucleotides and cardiac function following ischemia. Circulation 68, 389

210. St Cyr JA, Bianco RW, Schneider JR, et al. (1989) Enhanced high energy phosphate recovery with ribose infusion after global myocardial ischemia in a canine model. J Surg Res 46, 157-162.

211. Zimmer HG (1983) Normalization of depressed heart function in rats by ribose. Science 220, $81-82$.

212. Schneider J, St Cyr J \& Mahoney J (1985) Recovery of ATP and return of function after global ischemia. Circulation $\mathbf{7 2}$, Suppl., III_298.

213. Omran H, Illien S, MacCarter D, et al. (2003) D-Ribose improves diastolic function and quality of life in congestive heart failure patients: a prospective feasibility study. Eur $J$ Heart Fail 5, 615-619.

214. Vijay N, MacCarter D \& Shecterle LM (2008) D-Ribose benefits heart failure patients. J Med Food 11, 199-200.

215. Bayram M, St Cyr JA \& Abraham WT (2015) D-Ribose aids heart failure patients with preserved ejection fraction and diastolic dysfunction: a pilot study. Ther Adv Cardiovasc Dis 9, 56-65. 
216. Pliml W, von Arnim T, Stablein A, et al. (1992) Effects of ribose on exercise-induced ischaemia in stable coronary artery disease. Lancet 340, 507-510.

217. Perkowski D, Wagner S \& Marcus A (2007) D-Ribose improves cardiac indices in patients undergoing "off" pump coronary arterial revascularization. J Surg Res 137, 295.

218. Vance R, Einzig S \& Kreisler K (2000) D-Ribose maintains ejection fraction following aortic valve surgery. FASEB J 14, A 419 .

219. Nagatomo Y \& Tang WH (2015) Intersections between microbiome and heart failure: revisiting the gut hypothesis. J Card Fail 21, 973-980.

220. Sandek A, Bjarnason I, Volk HD, et al. (2012) Studies on bacterial endotoxin and intestinal absorption function in patients with chronic heart failure. Int J Cardiol 157, 80-85.

221. Krack A, Sharma R, Figulla HR, et al. (2005) The importance of the gastrointestinal system in the pathogenesis of heart failure. Eur Heart J 26, 2368-2374.

222. Organ CL, Otsuka H, Bhushan S, et al. (2016) Choline diet and its gut microbe derived metabolite, trimethylamine $\mathrm{N}$-oxide (TMAO), exacerbate pressure overload-induced heart failure. Circ Heart Fail 9, e002314.

223. Wang Z, Klipfell E, Bennett BJ, et al. (2011) Gut flora metabolism of phosphatidylcholine promotes cardiovascular disease. Nature 472, 57-63.

224. Koeth RA, Wang Z, Levison BS, et al. (2013) Intestinal microbiota metabolism of L-carnitine, a nutrient in red meat, promotes atherosclerosis. Nat Med 19, 576-585.

225. Wilson TWH, Zeneng W, Yiying F, et al. (2014) Prognostic value of elevated levels of intestinal microbe-generated metabolite trimethylamine- $N$-oxide in patients with heart failure. J Am Coll Cardiol 64, 1908-1914.

226. Shih DM, Wang Z, Lee R, et al. (2015) Flavin containing monooxygenase 3 exerts broad effects on glucose and lipid metabolism and atherosclerosis. J Lipid Res 56, 22-37.

227. Hartiala J, Bennett BJ, Tang WH, et al. (2014) Comparative genome-wide association studies in mice and humans for trimethylamine $N$-oxide, a proatherogenic metabolite of choline and L-carnitine. Arterioscler Thromb Vasc Biol 34, 1307-1313.

228. Costanza AC, Moscavitch SD, Faria Neto HC, et al. (2015) Probiotic therapy with Saccharomyces boulardii for heart failure patients: a randomized, double-blind, placebo-controlled pilot trial. Int J Cardiol 179, 348-350.

229. Gan XT, Ettinger G, Huang CX, et al. (2014) Probiotic administration attenuates myocardial hypertrophy and heart failure after myocardial infarction in the rat. Circ Heart Fail $\mathbf{7}$, 491-499.

230. Maier L, Pruteanu M, Kuhn M, et al. (2018) Extensive impact of non-antibiotic drugs on human gut bacteria. Nature $\mathbf{5 5 5}$, 623-628.

231. Jimenez K, Kulnigg-Dabsch S \& Gasche C (2015) Management of iron deficiency anemia. Gastroenterol Hepatol 11, 241-250.

232. Jankowska EA, Rozentryt P, Witkowska A, et al. (2011) Iron deficiency predicts impaired exercise capacity in patients with systolic chronic heart failure. J Card Fail 17, 899-906.

233. Tkaczyszyn M, Comín-Colet J, Voors AA, et al. (2017) Iron deficiency and red cell indices in patients with heart failure. Eur J Heart Fail 20, 114-122.

234. Van Aelst LNL, Abraham M, Sadoune M, et al. (2017) Iron status and inflammatory biomarkers in patients with acutely decompensated heart failure: Early in-hospital phase and 30-day follow-up. Eur J Heart Fail 19, 1075-1076.

235. Cohen-Solal A, Damy T, Hanon O, et al. (2014) High prevalence of iron deficiency in patients admitted for acute decompensated heart failure: a French study (CardioFer). J Am Coll Cardiol 63, Suppl., A779.
236. Okonko DO, Mandal AK, Missouris CG, et al. (2011) Disordered iron homeostasis in chronic heart failure: prevalence, predictors, and relation to anemia, exercise capacity, and survival. J Am Coll Cardiol 58, 1241-1251.

237. Ganz T \& Nemeth E (2006) Iron imports. IV. Hepcidin and regulation of body iron metabolism. Am J Physiol Gastrointest Liver Physiol 290, G199-G203.

238. Cunha GJL, Rocha BML \& Menezes Falcão L (2018) Iron deficiency in chronic and acute heart failure: a contemporary review on intertwined conditions. Eur J Intern Med 52, 1-7.

239. Hughes CM, Woodside JV, McGartland C, et al. (2012) Nutritional intake and oxidative stress in chronic heart failure. Nutr Metab Cardiovasc Dis 22, 376-382.

240. Lewis GD, Malhotra R, Hernandez AF, et al. (2017) Effect of oral iron repletion on exercise capacity in patients with heart failure with reduced ejection fraction and iron deficiency: the IRONOUT HF randomized clinical trial. JAMA $\mathbf{3 1 7}$, 1958-1966.

241. Drozd M, Jankowska EA, Banasiak W, et al. (2017) Iron therapy in patients with heart failure and iron deficiency: review of iron preparations for practitioners. Am J Cardiovasc Drugs 17, 183-201.

242. McDonagh T \& Macdougall IC (2015) Iron therapy for the treatment of iron deficiency in chronic heart failure: Intravenous or oral? Eur J Heart Fail 17, 248-262.

243. Bruckdorfer KR (2008) Antioxidants and CVD. Proc Nutr Soc 67, 214-222.

244. Wilcox BJ, Curb JD \& Rodriguez B (2008) Antioxidants in cardiovascular health and disease:key lessons from epidemiologic studies. Am J Cardiol 101, 75D-86D.

245. Djoussé L, Driver JA \& Gaziano JM (2009) Relation between modifiable lifestyle factors and lifetime risk of heart failure. JAMA 302, 394-400.

246. Levitan EB, Wolk A \& Mittleman MA (2009) Consistency with the DASH diet and incidence of heart failure. Arch Intern Med 169, 851-857.

247. Wang Y, Tuomilehto J, Jousilahti P, et al. (2011) Lifestyle factors in relation to heart failure among Finnish men and women. Circ Heart Fail 4, 607-612.

248. Pfister R, Sharp SJ, Luben R, et al. (2011) Plasma vitamin C predicts incident heart failure in men and women in European Prospective Investigation into Cancer and Nutrition-Norfolk Prospective Study. Am Heart J 162, 246-253.

249. Wannamethee SG, Bruckdorfer KR, Shaper AG, et al. (2013) Plasma vitamin C, but not vitamin $\mathrm{E}$, is associated with reduced risk of heart failure in older men. Circ Heart Fail 6, 647-654.

250. Song EK \& Kang SM (2018) Vitamin C deficiency, highsensitivity C-reactive protein, and cardiac event-free survival in patients with heart failure. J Cardiovasc Nurs 33, 6-12.

251. Ashor AW, Lara J, Mathers JC, et al. (2014) Effect of vitamin C on endothelial function in health and disease: a systematic review and meta-analysis of randomised controlled trials. Atherosclerosis 235, 9-20.

252. Plantinga Y, Ghiadoni L, Magagna A, et al. (2007) Supplementation with vitamins $\mathrm{C}$ and $\mathrm{E}$ improves arterial stiffness and endothelial function in essential hypertensive patients. Am J Hypertens 20, 392-397.

253. Bingham SA, Welch AA, McTaggart A, et al. (2001) Nutritional methods in the European Prospective Investigation of Cancer in Norfolk. Public Health Nutr 4, 847-858.

254. Spoelstra-de Man AME, Elbers PWG \& Oudemans-Van Straaten HM (2018) Vitamin C: should we supplement? Curr Opin Crit Care 24, 248-255.

255. Sawyer DB (2011) Oxidative stress in heart failure: what are we missing? Am J Med Sci 342, 120-124. 
256. Sen CK, Khanna S, Rink C, et al. (2007) Tocotrienols: the emerging face of natural vitamin E. Vitam Horm 76, 203-261.

257. Li F, Tan W, Kang Z, et al. (2010) Tocotrienol enriched palm oil prevents atherosclerosis through modulating the activities of peroxisome proliferators-activated receptors. Atherosclerosis 211, 278-282.

258. Rasool AH, Rahman AR, Yuen KH, et al. (2008) Arterial compliance and vitamin $\mathrm{E}$ blood levels with a self emulsifying preparation of tocotrienol rich vitamin E. Arch Pharm Res 31, 1212-1217.

259. Prasad K (2011) Tocotrienols and cardiovascular health. Curr Pharm Des 17, 2147-2154.

260. Chae CU, Albert CM, Moorthy MV, et al. (2012) Vitamin E supplementation and the risk of heart failure in women. Circ Heart Fail 5, 176-182.

261. Sesso HD, Buring JE, Christen WG, et al. (2008) Vitamins E and $\mathrm{C}$ in the prevention of cardiovascular disease in men: the Physicians' Health Study II randomized controlled trial. JAMA 300, 2123-2133.

262. Marchioli R, Levantesi G, Macchia A, et al. (2006) Vitamin E increases the risk of developing heart failure after myocardial infarction: results from the GISSI-Prevenzione Trial. J Cardiovasc Med 7, 347-350.

263. Lonn E, Bosch J, Yusuf S, et al. (2005) Effects of longterm vitamin E supplementation on cardiovascular events and cancer: a randomized controlled trial. JAMA 293, $1338-1347$

264. Finkel T \& Holbrook NJ (2000) Oxidants, oxidative stress and the biology of ageing. Nature $\mathbf{4 0 8}, 239-247$.

265. Deveraj D \& Jialei I (2005) Failure of vitamin E in clinical trials: is $\gamma$-tocopherol the answer? Nutr Rev 63, 290-293.

266. Hodge AM, Simpson JA, Fridman M, et al. (2009) Evaluation of an FFQ for assessment of antioxidant intake using plasma biomarkers in an ethnically diverse population. Public Health Nutr 12, 2438-2447.

267. Thurnham DI, Davies JA, Crump BJ, et al. (1986) The use of different lipids to express serum tocopherol: lipid ratios for the measurement of vitamin E status. Ann Clin Biochem 23, 514-520

268. Didonato JA, Aulak K, Huang Y, et al. (2014) Site-specific nitration of apolipoprotein A-I at tyrosine 166 is both abundant within human atherosclerotic plaque and dysfunctional. J Biol Chem 289, 10276-10292.

269. Holmberg CG \& Laurell CB (1948) Histaminolytic activity of a copper protein in serum. Nature 161, 236.

270. Alexanian I, Parissis J, Farmakis D, et al. (2014) Clinical and echocardiographic correlates of serum copper and zinc in acute and chronic heart failure. Clin Res Cardiol 103 938-949.

271. Ge K \& Yang G (1993) The epidemiology of selenium deficiency in the etiological study of endemic diseases in China. Am J Clin Nutr 57, Suppl. 2, 259S-263S.

272. De Lorgeril M, Salen P, Accominotti M, et al. (2001) Dietary and blood antioxidants in patients with chronic heart failure. Insights into the potential importance of selenium in heart failure. Eur J Heart Fail 3, 661-669.

273. Alehagen U, Alexander J \& Aaseth J (2016) Supplementation with selenium and coenzyme Q10 reduces cardiovascular mortality in elderly with low selenium status. a secondary analysis of a randomised clinical trial. PLOS ONE 11, e0157541.

274. Alehagen U, Johansson P, Björnstedt $\mathrm{M}$, et al. (2013) Cardiovascular mortality and N-terminal-proBNP reduced after combined selenium and coenzyme Q10 supplementation: a 5-year prospective randomized double-blind placebo-controlled trial among elderly Swedish citizens. Int J Cardiol 167, 1860-1866.

275. Da Cunha S, Albanesi Filho FM, da Cunha Bastos VL, et al. (2002) Thiamin, selenium, and copper levels in patients with idiopathic dilated cardiomyopathy taking diuretics. Arq Bras Cardiol 79, 454-465.

276. Malek F, Dvorak J, Jiresova E, et al. (2003) Difference of baseline serum copper levels between groups of patients with different one year mortality and morbidity and chronic heart failure. Cent Eur J Public Health 11, 198-201.

277. Keith ME, Walsh NA, Darling PB, et al. (2009) B-vitamin deficiency in hospitalized patients with heart failure. J Am Diet Assoc 109, 1406-1410.

278. Herzlich BC, Lichstein E, Schulhoff N, et al. (1996) Relationship among homocyst(e)ine, vitamin B-12 and cardiac disease in the elderly: association between vitamin B-12 deficiency and decreased left ventricular ejection fraction. J Nutr 126, Suppl., 1249S-1253S.

279. Witte KK, Desilva R, Chattopadhyay S, et al. (2004) Are hematinic deficiencies the cause of anemia in chronic heart failure? Am Heart J 147, 924-930.

280. Herrmann M, Muller S, Kindermann I, et al. (2007) Plasma B vitamins and their relation to the severity of chronic heart failure. Am J Clin Nutr 85, 117-123.

281. Andersson SE, Edvinsson ML \& Edvinsson L (2005) Reduction of homocysteine in elderly with heart failure improved vascular function and blood pressure control but did not affect inflammatory activity. Basic Clin Pharmacol Toxicol 97, 306-310.

282. Cicero AFG, Colletti A, Bajraktari G, et al. (2017) Lipid-lowering nutraceuticals in clinical practice: position paper from an International Lipid Expert Panel. Nutr Rev 75, 731-767.

283. Banach M, Patti AM, Giglio RV, et al. (2018) The role of nutraceuticals in statin intolerant patients. J Am Coll Cardiol $\mathbf{7 2}$, 96-118.

284. Sosnowska B, Penson P, Banach M (2017) The role of nutraceuticals in the prevention of cardiovascular disease. Cardiovasc Diagn Ther 7, Suppl. 1, S21-S31.

285. Patti AM, Al-Rasadi K, Giglio RV, et al. (2018) Natural approaches in metabolic syndrome management. Arch Med Sci 14, 422-441.

286. Bing RJ, Siegel A, Ungar I, et al. (1954) Metabolism of the human heart: II. Studies on fat, ketone and amino acid metabolism. Am J Med 16, 504-515.

287. Young LH, McNulty PH, Morgan C, et al. (1991) Myocardial protein turnover in patients with coronary artery disease. Effect of branched chain amino acid infusion. J Clin Invest 87, 554-560.

288. Rosenkranz ER, Okamoto F, Buckberg GD, et al. (1986) Safety of prolonged aortic clamping with blood cardioplegia. III. Aspartate enrichment of glutamate-blood cardioplegia in energy-depleted hearts after ischemic and reperfusion injury. I Thorac Cardiovasc Surg 91, 428-435.

289. Neubauer S, Horn M, Cramer M, et al. (1997) Myocardial phosphocreatine-to-ATP ratio is a predictor of mortality in patients with dilated cardiomyopathy. Circulation 96, 2190-2196.

290. Aquilani R, La Rovere MT, Corbellini D, et al. (2017) Plasma amino acid abnormalities in chronic heart failure. mechanisms, potential risks and targets in human myocardium metabolism. Nutrients 9, E1251.

291. Jeejeebhoy F, Keith M, Freeman M, et al. (2002) Nutritional supplementation with MyoVive repletes essential cardiac myocyte nutrients and reduces left ventricular size in patients with left ventricular dysfunction. Am Heart J 143 , 1092-1100. 


\section{Appendix}

International Lipid Expert Panel experts (alphabetically) are: Fahad Alnouri (Cardiovascular Prevention Unit, Adult Cardiology Department, Prince Sultan Cardiac Center, Riyadh, Saudi Arabia); Fabmy Amara (Unit of Diabetes \& Metabolism, Alexandria University, Alexandria, Egypt); Atanas G. Atanasov (Institute of Genetics and Animal Breeding of the Polish Academy of Sciences, Jastrzebiec, Poland; Department of Pharmacognosy, University of Vienna, Vienna, Austria; Institute of Neurobiology, Bulgarian Academy of Sciences, Sofia, Bulgaria); Gani Bajraktari (Institute of Public Health and Clinical Medicine, Umeå University, Umeå, Sweden; Clinic of Cardiology, University Clinical Centre of Kosova, Prishtina, Kosovo; Medical Faculty, University of Prishtina, Prishtina, Kosovo); Marcin A. Bartlomiejczyk (Department of Hypertension, Medical University of Lodz, Poland; Polish Mother's Memorial Hospital Research Institute (PMMHRI), Lodz, Poland); Bojko Bjelakovic (Clinic of Pediatrics, Clinical Center, Nis, Faculty of Medicine, University of Nis, Serbia); Eric Bruckert (Pitié-Salpêtrière Hospital and Sorbonne University, Cardio Metabolic Institute, Paris, France); Alberto Cafferata (Facultad de Medicina, Instituto Universitario de Ciencias de la Salud, Fundación H.A. Barceló, Argentina); Richard Ceska (Third Department of Medicine, Department of Endocrinology and Metabolism of the First Faculty of Medicine, Charles University and General University Hospital, Prague, Czech Republic); Xavier Collet (Institute of Metabolic and Cardiovascular Diseases, Inserm, Toulouse, France); Olivier Descamps (Department of Internal Medicine, Centres Hospitaliers Jolimont, Haine Saint-Paul, Belgium; Department of Cardiology, Cliniques Universitaires Saint-Luc, Bruxelles, Belgium); Nair Devaki (Department of Clinical Biochemistry, the Royal Free London NHS Foundation Trust, Pond Street, London, UK); Dragan Djuric (Institute of Medical Physiology "Richard Burian", Faculty of Medicine, University of Belgrade, Belgrade, Serbia); Ronen Durst (Cardiology Department, Hadassah Hebrew University Medical Center, Ein Kerem, Jerusalem, Israel); Marat V. Ezhov (National Cardiology Research Center, Moscow, Russia); Zlatko Fras (Preventive Cardiology Unit, Department of Vascular Medicine, Division of Medicine, University Medical Centre Ljubljana, Slovenia; Faculty of Medicine, University of Ljubljana, Ljubljana, Slovenia); Dan Gaita (Institutul de Boli Cardiovasculare, Universitatea de Medicina si Farmacie Victor Babes din Timisoara, Romania); Adrian V. Hernandez (Health Outcomes, Policy, and Evidence Synthesis (HOPES) Group, University of Connecticut/School of Pharmacy, Storrs, CT, USA; Vicerrectorado de Investigación, Universidad San Ignacio de Loyola (USIL), Lima, Peru); Steven R. Jones (The Johns Hopkins Ciccarone Center for the Prevention of Heart Disease, Baltimore, MD, USA); Jacek Jozwiak (Department of Family Medicine and Public Health, Faculty of Medicine, University of Opole, Opole, Poland); Nona Kakauridze (Department of Internal Medicine, Faculty of Medicine, Tbilisi State Medical University, Tbilisi, Georgia); Niki Katsiki (Second Department of Propaedeutic Internal Medicine, Medical School, Aristotle University of Thessaloniki, Hippocration Hospital, Thessaloniki, Greece);
Karam Kostner (Mater Hospital, University of Queensland, St Lucia, QLD, Australia); Raimondas Kubilius (Department of Rehabilitation, Medical Academy, Lithuanian University of Health Sciences, Kaunas, Lithuania); Gustavs Latkovskis (Institute of Cardiology and Regenerative Medicine, Faculty of Medicine, University of Latvia, Riga, Latvia; Pauls Stradins Clinical University Hospital, Riga, Latvia); G. B. John Mancini (Department of Medicine, Division of Cardiology, University of British Columbia, Vancouver, British Columbia, Canada); A. David Marais (Chemical Pathology Division of the Department of Pathology, University of Cape Town Health Science Faculty, Cape Town, South Africa); Seth S. Martin (Ciccarone Center for Prevention of Heart Disease, Division of Cardiology, Department of Medicine, Johns Hopkins University School of Medicine, Baltimore, MD, USA); Julio Acosta Martinez (Medico Cardiologo de la Policlinica Metropolitana, Caracas, Venezuela); Mohsen Mazidi (Department of Twin Research and Genetic Epidemiology, King's College London, St Thomas' Hospital, Strand, London, UK); Erkin Mirrakhimov (Kyrgyz State Medical Academy, named after Akhunbaev I.K., Bishkek, Kyrgyzstan); Andre R. Miserez (Diagene Research Institute, Reinach, Switzerland; President of Swiss Society of Familial Forms of Hypercholesterolemia (SSFH), Breitenbach, Switzerland; University of Basel, Basel, Switzerland); Olena Mitchenko (Dyslipidaemia Department, Institute of Cardiology AMS of Ukraine, Ukraine; Ukrainian Atherosclerosis Society); Patrick R. Moriarty (Division of Clinical Pharmacology, Division of Internal Medicine, University of Kansas Medical Center, Kansas City, KS, USA); Demosthenes B. Panagiotakos (School of Health Science and Education, Department of Nutrition and Dietetics, Harokopio University of Athens, Athens, Greece); György Paragh (Department of Internal Medicine, Faculty of Medicine, University of Debrecen, Debrecen, Hungary); Daniel Pella (1st Department of Internal Medicine, Faculty of Medicine, Pavol Jozef Safarik University, Košice, Slovakia); Peter Penson (School of Pharmacy and Biomolecular Sciences, Liverpool John Moores University, Liverpool, UK); Zaneta Petrulioniene (Vilnius University Faculty of Medicine, Vilnius, Lithuania; Vilnius University Hospital Santaros Klinikos, Vilnius, Lithuania); Matteo Pirro (Department of Medicine, University of Perugia, Perugia, Italy); Arman Postadzhiyan (Bulgarian Society of Cardiology, Medical University of Sofia, Sofia, Bulgaria); Raman Puri (Indraprastha Apollo Hospital, New Delhi, India); Ashraf Reda (Menoufia University NLI of FHSC, President of EAVA); Jemaa Riadh (Laboratory of Biochemistry, Faculty of Medicine of Tunis, Rabta Hospital, University of Tunis El Manar, Tunis, Tunisia); Dimitri Richter (Cardiac Department, Euroclinic, Athens, Greece); Manfredi Rizzo (Biomedical Department of Internal Medicine and Medical Specialties, University of Palermo, Palermo, Italy); Massimiliano Ruscica (Department of Pharmacological and Biomolecular Sciences, University of Milan, Milan, Italy); Naveed Sattar (Institute of Cardiovascular and Medical Sciences, University of Glasgow, Glasgow, UK); Maria-Corina Serban (Department of Functional Sciences, Discipline of Pathophysiology, "Victor Babes" University of Medicine and Pharmacy, Timisoara, Romania); Abdulla M. A Shehab (Medical Education 
Department, United Arab Emirates University, Al Ain, United Arab Emirates); Aleksandr B. Shek (Republican Specialised Center of Cardiology, Tashkent, Uzbekistan); Cesare R. Sirtori (Department of Pharmacological and Biomolecular Sciences, University of Milan, Milan, Italy); Claudia Stefanutti (Department of Molecular Medicine, Sapienza University of Rome, Rome, Italy); Tomasz Tomasik (Department of Family Medicine, Chair of Internal Medicine and Gerontology, Jagiellonian University Medical College, Krakow, Poland); Margus Viigimaa (Tallinn University of Technology, North Estonia Medical Centre, Tallinn, Estonia); Branislav Vobnout (Institute of Nutrition, Faculty of Nursing and Health Professional Studies and Coordination Centre for Familial Hyperlipoproteinemias, Slovak Medical University in Bratislava, Bratislava, Slovakia; Institute of Epidemiology,
School of Medicine, Comenius University, Bratislava, Slovakia); Michal Vrablik (1st Faculty of Medicine, Charles University and General University Hospital, Prague, Czech Republic); Nathan Wong (Department of Medicine, School of Medicine University of California, Irvine, CA, USA; Heart Disease Prevention Program, Division of Cardiology, University of California, Irvine, CA, USA); Hung-I Yeh (Department of Medicine, Mackay Medical College, Taipei, Taiwan; Cardiovascular Division, Department of Internal Medicine, MacKay Memorial Hospital, Taipei, Taiwan); Jiang Zhisheng (Institute of Cardiovascular Disease, University of South China, Hengyang, Hunan, China); Andreas Zirlik (University Heart Centre Freiburg University, Department of Cardiology and Angiology I, Faculty of Medicine, University of Freiburg, Freiburg, Germany). 